\title{
HOMOGENIZATION OF THE NON-STATIONARY STOKES EQUATIONS WITH PERIODIC VISCOSITY
}

\author{
Hi Jun Choe And Hyunseok Kim
}

\begin{abstract}
We study the periodic homogenization of the non-stationary Stokes equations. The fundamental homogenization theorem and corrector theorem are proved under a very general assumption on the viscosity coefficients and data. The proofs are based on a weak formulation suitable for an application of classical Tartar's method of oscillating test functions. Such a weak formulation is derived by adapting an argument in Teman's book [Navier-Stokes Equations: Theory and Numerical Analysis, NorthHolland, Amsterdam, 1984].
\end{abstract}

\section{Introduction}

The study of partial differential equations (PDEs) with $\varepsilon Y$-periodic coefficients, where $\varepsilon$ is a small parameter and $Y=[0,1]^{n}$ the unit torus, has been a classical subject in the theory of homogenization. Particularly well understood is the periodic homogenization of linear elliptic or parabolic PDEs of second order; the fundamental homogenization theorems and corrector theorems are proved in standard textbooks such as Bensoussan, Lions and Papanicolaou [2] and Sanchez-Palencia [12], etc. See also a recent book [5] by Cioranescu and Donato. The proofs of the homogenization theorems in $[2,5,12]$ are originally due to Tartar in the middle 1970s. The main difficulty in the homogenization of a linear elliptic PDE is to show the convergence, as $\varepsilon \rightarrow 0$, of products of two weakly convergent sequences appearing in the weak formulation of the PDE. This difficulty was overcome by Tartar using special test functions in the weak formulation. His test functions are given by products of a smooth cut-off function and $\varepsilon Y$-periodic solutions of the adjoint equation of the PDE.

Tartar's method of oscillating test functions was applied to the periodic homogenization of a system related to the stationary Stokes equations by Bensoussan, Lions and Papanicolaou [2]. The homogenization theorem in [2] for

Received December 10, 2007.

2000 Mathematics Subject Classification. 35B27, 35Q30, 76M50.

Key words and phrases. homogenization, periodic viscosity, non-stationary Stokes equations, oscillating test functions.

The second author was supported by the Korea Research Foundation Grant funded by the Korean Government (MOEHRD, Basic Research Promotion Fund) (KRF-2006-003-C00023). 
the Stokes-like system can be also extended to the stationary, isotropic Stokes equations with periodic viscosity $\mu^{\varepsilon}=\mu(\dot{\bar{\varepsilon}})$ :

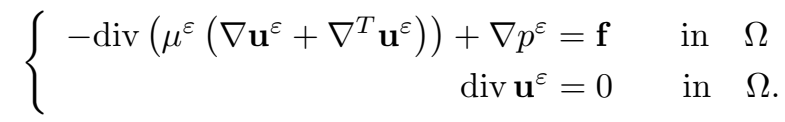

Here $\mathbf{u}^{\varepsilon}=\left(u_{1}^{\varepsilon}, \ldots, u_{n}^{\varepsilon}\right)$ and $p^{\varepsilon}$ denote the unknown velocity and pressure fields, respectively, of an isotropic, incompressible fluid in a bounded domain $\Omega$ of $\mathbb{R}^{n}$ and $\mathbf{f}$ is a given external force. Suppose that $\lambda \leq 2 \mu \leq M$ for some positive constants $\lambda$ and $M$. Then it follows from classical Korn's inequality that the bilinear form $a_{\mu}^{\varepsilon}$ defined by

$$
a_{\mu}^{\varepsilon}(\mathbf{v}, \mathbf{w})=\int_{\Omega} \mu^{\varepsilon}\left(\nabla \mathbf{v}+\nabla^{T} \mathbf{v}\right) \cdot\left(\nabla \mathbf{w}+\nabla^{T} \mathbf{w}\right) d x
$$

is coercive on both spaces $\mathbf{H}_{0}^{1}(\Omega)$ and $V$, where $\mathbf{H}_{0}^{1}(\Omega)$ and $V$ are the standard Sobolev spaces defined in a famous book [13] by Temam; see the end of this section for notations used throughout the paper. Hence the Lax-Milgram theorem implies that for each $\mathbf{f} \in V^{\prime}$, there exists a unique $\mathbf{u}^{\varepsilon} \in V$ such that

$$
a_{\mu}^{\varepsilon}\left(\mathbf{u}^{\varepsilon}, \mathbf{v}\right)=\langle\mathbf{f}, \mathbf{v}\rangle_{V^{\prime} \times V} \quad \text { for all } \quad \mathbf{v} \in V .
$$

But the weak formulation (2) is not suitable for a direct application of Tartar's method to the homogenization of (1) because only divergence-free vector fields are allowed as test functions in (2). A right weak formulation involves a pressure associated with $\mathbf{u}^{\varepsilon}$. To introduce a pressure in $L^{2}(\Omega)$, suppose in addition that $\mathbf{f} \in \mathbf{H}^{-1}(\Omega)$. Then since the mapping $\mathbf{v} \mapsto a_{\mu}^{\varepsilon}\left(\mathbf{u}^{\varepsilon}, \mathbf{v}\right)-\langle\mathbf{f}, \mathbf{v}\rangle_{\mathbf{H}^{-1}(\Omega) \times \mathbf{H}_{0}^{1}(\Omega)}$ is a bounded linear functional on $\mathbf{H}_{0}^{1}(\Omega)$ vanishing identically on $V$, it follows from a standard result in fluid mechanics (see [7] or [13]) that there exists a unique scalar field $p^{\varepsilon}$ in $L^{2}(\Omega) / \mathbb{R}$ such that

$$
a_{\mu}^{\varepsilon}\left(\mathbf{u}^{\varepsilon}, \mathbf{v}\right)-\left(p^{\varepsilon}, \operatorname{div} \mathbf{v}\right)_{L^{2}(\Omega)}=\langle\mathbf{f}, \mathbf{v}\rangle_{\mathbf{H}^{-1}(\Omega) \times \mathbf{H}_{0}^{1}(\Omega)}
$$

for all $\mathbf{v} \in \mathbf{H}_{0}^{1}(\Omega)$. Using this weak formulation, we can apply Tartar's method to deduce the homogenization theorem: $\left(\mathbf{u}^{\varepsilon}, p^{\varepsilon}\right)$ converges weakly to the unique weak solution $\left(\mathbf{u}^{0}, p^{0}\right) \in V \times L^{2}(\Omega) / \mathbb{R}$ of the homogenized equations

$$
\left\{\begin{aligned}
\mathbf{A}^{0} \mathbf{u}^{0}+\nabla p^{0}=\mathbf{f} & \text { in } \quad \Omega \\
\operatorname{div} \mathbf{u}^{0}=0 & \text { in } \Omega,
\end{aligned}\right.
$$

where the differential operator $\mathbf{A}^{0}=\left(A_{1}^{0}, \ldots, A_{n}^{0}\right)$ is defined by

$$
\mathbf{v}=\left(v_{1}, \ldots, v_{n}\right) \mapsto A_{j}^{0} \mathbf{v}=\left(\mathbf{A}^{0} \mathbf{v}\right)_{j}=-\frac{\partial}{\partial x_{\beta}}\left(\tilde{a}_{\alpha \beta}^{i j} \frac{\partial v_{i}}{\partial x_{\alpha}}\right)
$$

for some constants $\tilde{a}_{\alpha \beta}^{i j}, 1 \leq \alpha, \beta, i, j \leq n$, satisfying the symmetry and ellipticity conditions for the coefficients of linear elasticity

$$
\tilde{a}_{\alpha \beta}^{i j}=\tilde{a}_{i \beta}^{\alpha j}=\tilde{a}_{\alpha j}^{i \beta}=\tilde{a}_{\beta \alpha}^{j i}
$$


and

$$
\tilde{a}_{\alpha \beta}^{i j} \xi_{\alpha}^{i} \xi_{\beta}^{j}>0 \quad \text { for all } n \times n \text { symmetric matrices } \xi=\left(\xi_{\alpha}^{i}\right) .
$$

Here we adopt the summation convention for repeated indices. The corrector theorem then can be deduced from the homogenization theorem and energy identities by following classical arguments in $[2,5]$.

We have outlined proofs of the fundamental theorems on the homogenization of the stationary Stokes equations (1). But our main concern in the paper is a more difficult problem of the periodic homogenization of the non-stationary Stokes equations in a quite general setting. To be precise, we are interested in analyzing the asymptotic behavior, as $\varepsilon \rightarrow 0$, of the weak solution $\mathbf{u}^{\varepsilon}$ to the following initial boundary value problem

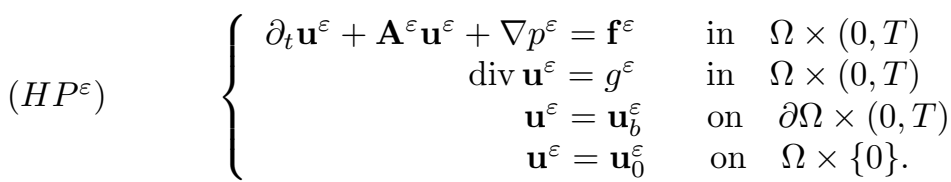

Here $\Omega$ is a bounded domain in $\mathbb{R}^{n}, n \geq 2$, with smooth boundary $\partial \Omega, T$ is a finite positive number and the vector or scalar fields $\mathbf{f}^{\varepsilon}, g^{\varepsilon}, \mathbf{u}_{b}^{\varepsilon}$ and $\mathbf{u}_{0}^{\varepsilon}$ are known data. Moreover, the differential operator $\mathbf{A}^{\varepsilon}=\left(A_{1}^{\varepsilon}, \ldots, A_{n}^{\varepsilon}\right)$ is defined by

$$
\mathbf{v}=\left(v_{1}, \ldots, v_{n}\right) \mapsto A_{j}^{\varepsilon} \mathbf{v}=\left(\mathbf{A}^{\varepsilon} \mathbf{v}\right)_{j}=-\frac{\partial}{\partial x_{\beta}}\left(a_{\alpha \beta}^{i j}\left(\frac{\mathbf{x}}{\varepsilon}\right) \frac{\partial v_{i}}{\partial x_{\alpha}}\right)
$$

for some functions $a_{\alpha \beta}^{i j}, 1 \leq \alpha, \beta, i, j \leq n$, in $\mathbb{R}^{n}$ satisfying the following hypotheses:

$$
\begin{array}{lll}
\text { (4) } & \text { (boundedness) } & M=\sup _{1 \leq \alpha, \beta, i, j \leq n}\left\|a_{\alpha \beta}^{i j}\right\|_{L^{\infty}\left(\mathbb{R}^{n}\right)}<\infty, \\
\text { (5) (periodicity) } & a_{\alpha \beta}^{i j}\left(\mathbf{y}+\mathbf{e}^{k}\right)=a_{\alpha \beta}^{i j}(\mathbf{y}) \quad(1 \leq k \leq n), \\
\text { (6) (symmetry) } & a_{\alpha \beta}^{i j}(\mathbf{y})=a_{i \beta}^{\alpha j}(\mathbf{y})=a_{\alpha j}^{i \beta}(\mathbf{y}),
\end{array}
$$

and

$$
\text { (ellipticity) } \quad a_{\alpha \beta}^{i j}(\mathbf{y}) \xi_{\alpha}^{i} \xi_{\beta}^{j} \geq \lambda|\xi|^{2}
$$

for a.e. $\mathbf{y} \in \mathbb{R}^{n}$ and all $n \times n$-symmetric matrices $\xi=\left(\xi_{\alpha}^{i}\right)$, where $M, \lambda$ are positive constants and $\left\{\mathbf{e}^{k}\right\}_{1 \leq k \leq n}$ is the canonical basis of $\mathbb{R}^{n} ; e_{i}^{k}=\delta_{k i}$ equals 1 if $k=i$ and 0 otherwise. Note that the conditions (6) and (7) hold for the isotropic case that

$$
a_{\alpha \beta}^{i j}(\mathbf{y})=\mu(\mathbf{y})\left(\delta_{\alpha \beta} \delta_{i j}+\delta_{\alpha j} \delta_{i \beta}\right) \quad \text { and } \quad 0<\lambda \leq 2 \mu(\mathbf{y})
$$

for a.e. $\mathbf{y} \in \mathbb{R}^{n}$

The main purpose of the paper is to prove the following fundamental theorems on the homogenization of $\left(H P^{\varepsilon}\right)$ : 
The homogenization theorem If the data $\mathbf{f}^{\varepsilon}, g^{\varepsilon}, \mathbf{u}_{b}^{\varepsilon}$ and $\mathbf{u}_{0}^{\varepsilon}$ satisfy some general hypotheses, then the unique weak solution $\mathbf{u}^{\varepsilon}$ to the problem $\left(H P^{\varepsilon}\right)$ converges weakly to the unique weak solution $\mathbf{u}^{0}$ to the homogenized problem $\left(H P^{0}\right)$ as $\varepsilon \rightarrow 0$.

The corrector theorem Moreover, if $\mathbf{u}^{0}$ is sufficiently regular, then there exists a corrector $\mathbf{u}^{1}\left(\mathbf{x}, t, \frac{\mathbf{x}}{\varepsilon}\right)$ such that $\mathbf{u}^{\varepsilon}-\varepsilon \mathbf{u}^{1}$ converges strongly to $\mathbf{u}^{0}$ as $\varepsilon \rightarrow 0$.

The precise statements of the homogenization and corrector theorems are given in Sections 4 and 5, respectively.

Our proofs of the fundamental theorems rely on Tartar's method of oscillating test functions. To easily explain key ideas of the proofs, we assume for the time being that

(8) $\quad \mathbf{f}^{\varepsilon}=\mathbf{0}, \quad g^{\varepsilon}=0, \quad \mathbf{u}_{b}^{\varepsilon}=\mathbf{0} \quad$ and $\quad \mathbf{u}_{0}^{\varepsilon}=\mathbf{u}_{0} \in H \quad$ (independent of $\varepsilon$ ).

Then a standard argument allows us to deduce the existence of a unique vector field $\mathbf{u}^{\varepsilon}$ in $\mathbf{V}_{T} \equiv L^{2}(0, T ; V) \cap W^{1,2}\left(0, T ; V^{\prime}\right)$ such that

$$
\mathbf{u}^{\varepsilon}(0)=\mathbf{u}_{0} \quad \text { and } \quad\left\langle\partial_{t} \mathbf{u}^{\varepsilon}(t), \mathbf{v}\right\rangle_{V^{\prime} \times V}+a^{\varepsilon}\left(\mathbf{u}^{\varepsilon}(t), \mathbf{v}\right)=0
$$

for a.e. $t \in(0, T)$ and all $\mathbf{v} \in V$, where the bilinear form $a^{\varepsilon}$ is defined by

$$
a^{\varepsilon}(\mathbf{v}, \mathbf{w})=\int_{\Omega} a_{\alpha \beta}^{i j}\left(\frac{\mathbf{x}}{\varepsilon}\right) \frac{\partial v_{i}}{\partial x_{\alpha}} \frac{\partial w_{j}}{\partial x_{\beta}} d x .
$$

Recall that the introduction of a pressure in $L^{2}(\Omega)$ enables us to derive a right weak formulation in the application of Tartar's method to the stationary Stokes equations. But in case of the non-stationary Stokes equations, the lack of the regularity of $\partial_{t} \mathbf{u}^{\varepsilon}$ prevents us from concluding that the associated pressure $p^{\varepsilon}$ is an $L^{2}$-function in $\Omega \times(0, T)$; it would follows that $p^{\varepsilon} \in L^{2}\left(0, T ; L^{2}(\Omega)\right)$ if $\partial_{t} \mathbf{u}^{\varepsilon} \in$ $L^{2}\left(0, T ; \mathbf{H}^{-1}(\Omega)\right)$ could be shown. In fact, $p^{\varepsilon}$ is just a distribution in $\Omega \times(0, T)$, which makes the non-stationary problem more difficult than the stationary one. To overcome this difficulty, we derive a new weak formulation by adapting an argument in Teman's book [13, Section 3.1]. The crucial ingredient is the well-known fact that if $\mathbf{v}, \mathbf{w} \in \mathbf{V}_{T}$, then

$$
\frac{d}{d t}(\mathbf{v}(t), \mathbf{w}(t))_{\mathbf{L}^{2}(\Omega)}=\left\langle\partial_{t} \mathbf{v}(t), \mathbf{w}(t)\right\rangle_{V^{\prime} \times V}+\left\langle\partial_{t} \mathbf{w}(t), \mathbf{v}(t)\right\rangle_{V^{\prime} \times V}
$$

for a.e. $t \in[0, T]$. For a standard proof, see [5, Section 3.5] for instance. As an immediate consequence of (11), we have

$$
\left(\mathbf{u}^{\varepsilon}(t)-\mathbf{u}^{\varepsilon}(0), \mathbf{v}\right)_{\mathbf{L}^{2}(\Omega)}=\int_{0}^{t}\left\langle\partial_{t} \mathbf{u}^{\varepsilon}(\tau), \mathbf{v}\right\rangle_{V^{\prime} \times V} d \tau
$$

for all $t \in[0, T]$ and $\mathbf{v} \in V$. Combining this and (9), we deduce that

$$
\left(\mathbf{u}^{\varepsilon}(t)-\mathbf{u}_{0}, \mathbf{v}\right)_{\mathbf{L}^{2}(\Omega)}+\int_{0}^{t} a^{\varepsilon}\left(\mathbf{u}^{\varepsilon}(\tau), \mathbf{v}\right) d \tau=0
$$


for all $t \in[0, T]$ and $\mathbf{v} \in V$, which then implies the existence of a unique scalar $P^{\varepsilon} \in C\left([0, T] ; L^{2}(\Omega) / \mathbb{R}\right)$ such that

$$
\left(\mathbf{u}^{\varepsilon}(t)-\mathbf{u}_{0}, \mathbf{v}\right)_{\mathbf{L}^{2}(\Omega)}-\left(P^{\varepsilon}(t), \operatorname{div} \mathbf{v}\right)_{L^{2}(\Omega)}+\int_{0}^{t} a^{\varepsilon}\left(\mathbf{u}^{\varepsilon}(\tau), \mathbf{v}\right) d \tau=0
$$

for all $t \in[0, T]$ and $\mathbf{v} \in \mathbf{H}_{0}^{1}(\Omega)$. Using this weak formulation, we can apply Tartar's method to deduce the homogenization theorem: $\mathbf{u}^{\varepsilon}$ converges weakly in $\mathbf{V}_{T}$ to a unique vector field $\mathbf{u}^{0}$ in $\mathbf{V}_{T}$ such that

$$
\left(\mathbf{u}^{0}(t)-\mathbf{u}_{0}, \mathbf{v}\right)_{\mathbf{L}^{2}(\Omega)}+\int_{0}^{t} a^{0}\left(\mathbf{u}^{0}(\tau), \mathbf{v}\right) d \tau=0
$$

for all $t \in[0, T]$ and $\mathbf{v} \in V$, where $a^{0}$ is a bilinear form on $\mathbf{H}^{1}(\Omega)$ defined later by (39) and (44). On the other hand, the identity (11) with $\mathbf{v}=\mathbf{w}$ enables us to derive the energy identities of $\mathbf{u}^{\varepsilon}$ and $\mathbf{u}^{0}$ from (9) and a differential form of (13). Using the homogenization theorem and the energy identities, we can then show that

$$
\frac{1}{2}\left\|\mathbf{u}^{\varepsilon}(t)-\mathbf{u}^{0}(t)\right\|_{\mathbf{L}^{2}(\Omega)}+\int_{0}^{t} a^{\varepsilon}\left(\mathbf{u}^{\varepsilon}(\tau), \mathbf{u}^{\varepsilon}(\tau)\right) d \tau \rightarrow \int_{0}^{t} a^{0}\left(\mathbf{u}^{0}(\tau), \mathbf{u}^{0}(\tau)\right) d \tau
$$

as $\varepsilon \rightarrow 0$ for each $t \in[0, T]$. This convergence is actually uniform on $[0, T]$ thanks to the Ascoli-Arzelà theorem. Hence adapting the arguments in [5], we can deduce the corrector theorem: $\mathbf{u}^{\varepsilon} \rightarrow \mathbf{u}^{0}$ strongly in $C\left([0, T] ; \mathbf{L}^{2}(\Omega)\right)$ and if $\mathbf{u}^{0}$ is sufficiently regular, then there is a corrector $\mathbf{u}^{1}\left(\mathbf{x}, t, \frac{\mathbf{x}}{\varepsilon}\right)$ such that $\mathbf{u}^{\varepsilon}-\varepsilon \mathbf{u}^{1} \rightarrow \mathbf{u}^{0}$ strongly in $L^{2}\left(0, T ; \mathbf{H}^{1}(\Omega)\right)$.

We have provided the key ideas of our proofs of the fundamental theorems of the homogenization of $\left(H P^{\varepsilon}\right)$ with the data satisfying (8). The complete proofs of the theorems in their full generality will be provided in Sections 4 and 5. In Section 4, we prove the homogenization theorem, Theorem 7 , by applying Tartar's method of oscillating test functions. The suitable weak formulation and energy identity are derived in Section 2 where we also establish the wellposedness of the problem $\left(H P^{\varepsilon}\right)$ with $\varepsilon>0$ fixed. We remark that the identity (11) is the crucial ingredient of the derivations of weak formulation and energy identity and of the proof of the uniform convergence of $\mathbf{u}^{\varepsilon}$ as well. In Section 3, we apply the standard multi-scale method to derive the homogenized problem $\left(H P^{0}\right)$, that is, the limiting problem of $\left(H P^{\varepsilon}\right)$ as $\varepsilon \rightarrow 0$. The well-posedness of $\left(H P^{0}\right)$ is also established. The final section, Section 5 , is devoted to proving the corrector theorem, Theorem 9 . The proof is based on the homogenization theorem and energy identities. Another technical tools are classical Korn's inequality for vector fields in $\mathbf{H}^{1}(\Omega)$ and a $L^{r}$-regularity result, Lemma 11 , on weak solutions of the stationary Stokes equations with $L^{\infty}$-viscosity coefficients. For completeness, we provide a detailed proof of Lemma 11 using the reverse Hölder inequality.

We finish this introductory section with explaining the notations and convection used throughout this paper. 
Notations throughout the paper. (i) For the function spaces, we adopt the following notations in Teman's book [13]:

$1<r<\infty, \quad L^{r}(\Omega) / \mathbb{R}=\left\{h \in L^{r}(\Omega): \int_{\Omega} h d x=0\right\}, \quad \mathbf{L}^{r}(\Omega)=\left\{L^{r}(\Omega)\right\}^{n}$,

$\mathbf{W}^{2, r}(\Omega)=\left\{W^{2, r}(\Omega)\right\}^{n}, \mathbf{H}^{1}(\Omega)=\left\{H^{1}(\Omega)\right\}^{n}, \mathbf{H}^{\frac{1}{2}}(\partial \Omega)=\left\{\left.\mathbf{h}\right|_{\partial \Omega}: \mathbf{h} \in \mathbf{H}^{1}(\Omega)\right\}$,

$\mathcal{D}(\Omega)=C_{0}^{\infty}(\Omega), \mathbf{H}_{0}^{1}(\Omega)=\overline{\{\mathcal{D}(\Omega)\}^{n}} \mathbf{H}^{1}(\Omega), \mathbf{H}^{-1}(\Omega)=\left[\mathbf{H}_{0}^{1}(\Omega)\right]^{\prime}$,

$\mathcal{V} \equiv\left\{\mathbf{h} \in\{\mathcal{D}(\Omega)\}^{n}: \operatorname{div} \mathbf{h}=0 \quad\right.$ in $\left.\Omega\right\}, \quad H=\overline{\mathcal{V}}^{\mathbf{L}^{2}(\Omega)} \quad$ and $\quad V=\overline{\mathcal{V}}^{\mathbf{H}^{1}(\Omega)}$.

(ii) The dual paring of any Banach space $X$ and its dual $X^{\prime}$ is denoted by $\langle\cdot, \cdot\rangle_{X^{\prime} \times X}$ or simply $\langle\cdot, \cdot\rangle$. Similarly, the inner product of any Hilbert space $X$ is denoted by $(\cdot, \cdot)_{X}$ or simply $(\cdot, \cdot)$.

(iii) We denote by $C$ a generic positive constant depending only on $n, \lambda, M, T$ and $\Omega$, etc. but not on $\varepsilon$.

\section{The well-posedness of $\left(H P^{\varepsilon}\right)$}

In this section, after deriving a suitable weak formulation, we prove the wellposedness of the problem $\left(H P^{\varepsilon}\right)$ with the data $\mathbf{f}^{\varepsilon}, g^{\varepsilon}, \mathbf{u}_{b}^{\varepsilon}$ and $\mathbf{u}_{0}^{\varepsilon}$ satisfying the regularity

$$
\begin{gathered}
\mathbf{f}^{\varepsilon} \in L^{2}\left(0, T ; \mathbf{H}^{-1}(\Omega)\right), \quad g^{\varepsilon} \in L^{2}\left(0, T ; L^{2}(\Omega)\right), \\
\mathbf{u}_{b}^{\varepsilon} \in L^{2}\left(0, T ; \mathbf{H}^{\frac{1}{2}}(\partial \Omega)\right) \quad \text { and } \quad \mathbf{u}_{0}^{\varepsilon} \in \mathbf{L}^{2}(\Omega) .
\end{gathered}
$$

First of all, to assure the existence of weak solutions with some regularity, we impose a compatibility condition on $g^{\varepsilon}, \mathbf{u}_{b}^{\varepsilon}$ and $\mathbf{u}_{0}^{\varepsilon}$. Assume that there exists a vector field

$$
\mathbf{G}^{\varepsilon} \in \mathbf{W}_{T} \equiv L^{2}\left(0, T ; \mathbf{H}^{1}(\Omega)\right) \cap W^{1,2}\left(0, T ; \mathbf{H}^{-1}(\Omega)\right)
$$

such that

(16) $\operatorname{div} \mathbf{G}^{\varepsilon}=g^{\varepsilon}$ in $\Omega \times(0, T),\left.\quad \mathbf{G}^{\varepsilon}\right|_{\partial \Omega \times(0, T)}=\mathbf{u}_{b}^{\varepsilon} \quad$ and $\quad \mathbf{u}_{0}^{\varepsilon}-\mathbf{G}^{\varepsilon}(0) \in H$.

Here it should be noticed that the space $\mathbf{W}_{T}$ can be continuously embedded into $C\left([0, T] ; \mathbf{L}^{2}(\Omega)\right)$ and $\mathbf{G}^{\varepsilon}(0)$ is a well-defined vector field belonging to $\mathbf{L}^{2}(\Omega)$.

Introducing a new unknown $\mathbf{v}^{\varepsilon}=\mathbf{u}^{\varepsilon}-\mathbf{G}^{\varepsilon}$, we can then reduce $\left(H P^{\varepsilon}\right)$ to an equivalent problem

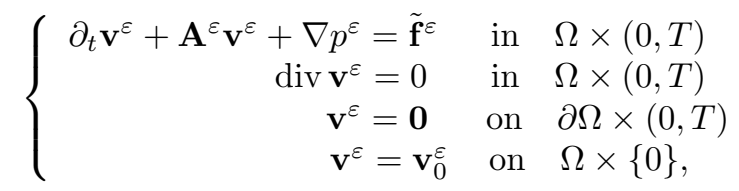

where $\tilde{\mathbf{f}}^{\varepsilon}=\mathbf{f}^{\varepsilon}-\partial_{t} \mathbf{G}^{\varepsilon}-\mathbf{A}^{\varepsilon} \mathbf{G}^{\varepsilon}$ and $\mathbf{v}_{0}^{\varepsilon}=\mathbf{u}_{0}^{\varepsilon}-\mathbf{G}^{\varepsilon}(0)$ satisfy

$$
\begin{gathered}
\tilde{\mathbf{f}}^{\varepsilon} \in L^{2}\left(0, T ; \mathbf{H}^{-1}(\Omega)\right), \quad \mathbf{v}_{0}^{\varepsilon} \in H, \\
\langle\tilde{\mathbf{f}}, \mathbf{w}\rangle=\left\langle\mathbf{f}^{\varepsilon}-\partial_{t} \mathbf{G}^{\varepsilon}, \mathbf{w}\right\rangle-a^{\varepsilon}\left(\mathbf{G}^{\varepsilon}, \mathbf{w}\right) \quad \text { for all } \quad \mathbf{w} \in \mathbf{H}_{0}^{1}(\Omega)
\end{gathered}
$$


and

$$
\begin{aligned}
& \left\|\tilde{\mathbf{f}}^{\varepsilon}\right\|_{L^{2}\left(0, T ; \mathbf{H}^{-1}(\Omega)\right)}+\left\|\mathbf{v}_{0}^{\varepsilon}\right\|_{\mathbf{L}^{2}(\Omega)} \\
\leq & C\left(\left\|\mathbf{f}^{\varepsilon}\right\|_{L^{2}\left(0, T ; \mathbf{H}^{-1}(\Omega)\right)}+\left\|\mathbf{u}_{0}^{\varepsilon}\right\|_{\mathbf{L}^{2}(\Omega)}+\left\|\mathbf{G}^{\varepsilon}\right\|_{\mathbf{W}_{T}}\right) .
\end{aligned}
$$

Recall from (10) that $a^{\varepsilon}$ is the bilinear form on $\mathbf{H}^{1}(\Omega)$ defined by

$$
a^{\varepsilon}(\mathbf{v}, \mathbf{w})=\int_{\Omega} a_{\alpha \beta}^{i j}\left(\frac{\mathbf{x}}{\varepsilon}\right) \frac{\partial v_{i}}{\partial x_{\alpha}} \frac{\partial w_{j}}{\partial x_{\beta}} d x \quad \text { for } \quad \mathbf{v}, \mathbf{w} \in \mathbf{H}^{1}(\Omega) .
$$

On the other hand, in view of (6) and (7), we have

$$
a_{\alpha \beta}^{i j}(\mathbf{y}) \xi_{\alpha}^{i} \xi_{\beta}^{j}=\frac{1}{4} a_{\alpha \beta}^{i j}(\mathbf{y})\left(\xi_{\alpha}^{i}+\xi_{i}^{\alpha}\right)\left(\xi_{\beta}^{j}+\xi_{j}^{\beta}\right) \geq \frac{\lambda}{4}\left|\xi_{\alpha}^{i}+\xi_{i}^{\alpha}\right|^{2}
$$

for a.e. $\mathbf{y} \in \mathbb{R}^{n}$ and all $n \times n$-matrices $\xi=\left(\xi_{\alpha}^{i}\right)$. Hence it follows from (4) and (18) that $a^{\varepsilon}$ is a bounded, coercive form on $V$ and thus the Lax-Milgram theorem is applicable. In the simplistic case that $\mathbf{A}^{\varepsilon}=-\Delta$ (the Laplacian), the unique solvability of the reduced problem (17) has been established by several classical methods, for instance, a semi-discrete Galerkin method in Teman's book [13]. Following exactly the same arguments as in [13], we can prove the existence of a unique weak solution $\mathbf{v}^{\varepsilon}$ to the problem (17): by a weak solution to (17), we mean a vector field $\mathbf{v}^{\varepsilon}$ in $\Omega \times(0, T)$ such that

$$
\mathbf{v}^{\varepsilon} \in \mathbf{V}_{T} \equiv L^{2}(0, T ; V) \cap W^{1,2}\left(0, T ; V^{\prime}\right), \quad \mathbf{v}^{\varepsilon}(0)=\mathbf{v}_{0}^{\varepsilon}
$$

and

$$
\left\langle\partial_{t} \mathbf{v}^{\varepsilon}(t), \mathbf{w}\right\rangle+a^{\varepsilon}\left(\mathbf{v}^{\varepsilon}(t), \mathbf{w}\right)=\left\langle\tilde{\mathbf{f}}^{\varepsilon}(t), \mathbf{w}\right\rangle
$$

for a.e. $t \in(0, T)$ and all $\mathbf{w} \in V$. It should be also noted that the initial condition makes sense because $\mathbf{V}_{T}$ is continuously embedded into $C([0, T] ; H)$; see [13]. From (11), we immediately deduce that

$$
(\mathbf{v}(t)-\mathbf{v}(0), \mathbf{w})_{\mathbf{L}^{2}(\Omega)}=\int_{0}^{t}\left\langle\partial_{t} \mathbf{v}(\tau), \mathbf{w}\right\rangle d \tau
$$

for all $t \in[0, T]$ and $\mathbf{w} \in V$. Hence the integration of (19) in time leads to an equivalent weak formulation:

$$
\begin{gathered}
\mathbf{v}^{\varepsilon} \in \mathbf{V}_{T} \\
\left(\mathbf{v}^{\varepsilon}(t)-\mathbf{v}_{0}^{\varepsilon}, \mathbf{w}\right)_{\mathbf{L}^{2}(\Omega)}+\int_{0}^{t} a^{\varepsilon}\left(\mathbf{v}^{\varepsilon}(\tau), \mathbf{w}\right) d \tau=\int_{0}^{t}\left\langle\tilde{\mathbf{f}}^{\varepsilon}(\tau), \mathbf{w}\right\rangle d \tau
\end{gathered}
$$

for all $t \in[0, T]$ and $\mathbf{w} \in V$. Moreover, using the identity (11) with $\mathbf{w}=\mathbf{v}$, we easily deduce from (19) that

$$
\begin{aligned}
& \left\|\mathbf{v}^{\varepsilon}(t)\right\|_{\mathbf{L}^{2}(\Omega)}^{2}+2 \int_{0}^{t} a^{\varepsilon}\left(\mathbf{v}^{\varepsilon}(\tau), \mathbf{v}^{\varepsilon}(\tau)\right) d \tau \\
= & \left\|\mathbf{v}_{0}^{\varepsilon}\right\|_{\mathbf{L}^{2}(\Omega)}^{2}+2 \int_{0}^{t}\left\langle\tilde{\mathbf{f}}^{\varepsilon}(\tau), \mathbf{v}^{\varepsilon}(\tau)\right\rangle d \tau
\end{aligned}
$$


for all $t \in[0, T]$. An immediate consequence of the energy identity (22) is the following a priori estimate of $\mathbf{v}^{\varepsilon}$ :

$$
\sup _{0 \leq t \leq T}\left\|\mathbf{v}^{\varepsilon}\right\|_{\mathbf{L}^{2}(\Omega)}^{2}+\int_{0}^{T}\left\|\mathbf{v}^{\varepsilon}\right\|_{\mathbf{H}^{1}(\Omega)}^{2} d t \leq C\left(\left\|\mathbf{v}_{0}^{\varepsilon}\right\|_{\mathbf{L}^{2}(\Omega)}^{2}+\int_{0}^{T}\left\|\tilde{\mathbf{f}}^{\varepsilon}\right\|_{\mathbf{H}^{-1}(\Omega)}^{2} d t\right) .
$$

Converting back into the original variables and combining all the above estimates, we have proved the following well-posedness result for $\left(H P^{\varepsilon}\right)$.

Theorem 1. Let $\varepsilon>0$ be given. Then for any data $\mathbf{f}^{\varepsilon}, g^{\varepsilon}, \mathbf{u}_{b}^{\varepsilon}$ and $\mathbf{u}_{0}^{\varepsilon}$ satisfying the conditions (14)-(16), there exists a unique weak solution $\mathbf{u}^{\varepsilon}$ to the problem $\left(H P^{\varepsilon}\right)$ in the sense that

$$
\mathbf{u}^{\varepsilon}-\mathbf{G}^{\varepsilon} \in \mathbf{V}_{T}
$$

and

$$
\left(\mathbf{u}^{\varepsilon}(t)-\mathbf{u}_{0}^{\varepsilon}, \mathbf{w}\right)_{\mathbf{L}^{2}(\Omega)}+\int_{0}^{t} a^{\varepsilon}\left(\mathbf{u}^{\varepsilon}(\tau), \mathbf{w}\right) d \tau=\int_{0}^{t}\left\langle\mathbf{f}^{\varepsilon}(\tau), \mathbf{w}\right\rangle d \tau
$$

for all $t \in[0, T]$ and $\mathbf{w} \in V$. Moreover, the solution $\mathbf{u}^{\varepsilon}$ satisfies

$$
\begin{aligned}
& \sup _{0 \leq t \leq T}\left\|\mathbf{u}^{\varepsilon}(t)\right\|_{\mathbf{L}^{2}(\Omega)}^{2}+\int_{0}^{T}\left(\left\|\mathbf{u}^{\varepsilon}(t)\right\|_{\mathbf{H}^{1}(\Omega)}^{2}+\left\|\partial_{t} \mathbf{u}^{\varepsilon}(t)\right\|_{V^{\prime}}^{2}\right) d t \\
\leq & C\left(\left\|\mathbf{u}_{0}^{\varepsilon}\right\|_{\mathbf{L}^{2}(\Omega)}^{2}+\left\|\mathbf{G}^{\varepsilon}\right\|_{\mathbf{W}_{T}}^{2}+\int_{0}^{T}\left\|\mathbf{f}^{\varepsilon}(t)\right\|_{\mathbf{H}^{-1}(\Omega)}^{2} d t\right)
\end{aligned}
$$

for some positive constant $C=C(M, \lambda, \Omega)$, independent of $\varepsilon$.

\section{A formal asymptotic analysis and the homogenized problem}

In this section, we find and analyze the homogenized problem, that is, the limiting problem of $\left(H P^{\varepsilon}\right)$ as $\varepsilon \rightarrow 0$, by performing the multi-scale method. Suppose that $\left(\mathbf{u}^{\varepsilon}, p^{\varepsilon}\right)$ has a formal asymptotic expansion of the form

$$
\mathbf{u}^{\varepsilon}(\mathbf{x}, t)=\mathbf{u}^{0}\left(\mathbf{x}, t, \frac{\mathbf{x}}{\varepsilon}\right)+\varepsilon \mathbf{u}^{1}\left(\mathbf{x}, t, \frac{\mathbf{x}}{\varepsilon}\right)+\varepsilon^{2} \mathbf{u}^{2}\left(\mathbf{x}, t, \frac{\mathbf{x}}{\varepsilon}\right)+\cdots
$$

and

$$
p^{\varepsilon}(\mathbf{x}, t)=p^{0}\left(\mathbf{x}, t, \frac{\mathbf{x}}{\varepsilon}\right)+\varepsilon p^{1}\left(\mathbf{x}, t, \frac{\mathbf{x}}{\varepsilon}\right)+\cdots,
$$

where $\mathbf{u}^{k}$ and $p^{k}$ are functions in $\Omega \times[0, T] \times \mathbb{R}^{n}$ such that $\mathbf{u}^{k}(\mathbf{x}, t, \mathbf{y})$ and $p^{k}(\mathbf{x}, t, \mathbf{y})$ are periodic in $\mathbf{y}$ for each $(\mathbf{x}, t) \in \Omega \times[0, T]$. Assume for simplicity that $\mathbf{f}^{\varepsilon}=\mathbf{f}^{0}$ and $g^{\varepsilon}=g^{0}$.

By a direct calculation, we have

$$
\begin{gathered}
\nabla p^{k}\left(\mathbf{x}, t, \frac{\mathbf{x}}{\varepsilon}\right)=\left(\varepsilon^{-1} \nabla_{\mathbf{y}} p^{k}+\nabla_{\mathbf{x}} p^{k}\right)\left(\mathbf{x}, t, \frac{\mathbf{x}}{\varepsilon}\right) \\
\operatorname{div} \mathbf{u}^{k}\left(\mathbf{x}, t, \frac{\mathbf{x}}{\varepsilon}\right)=\left(\varepsilon^{-1} \operatorname{div}_{\mathbf{y}} \mathbf{u}^{k}+\operatorname{div}_{\mathbf{x}} \mathbf{u}^{k}\right)\left(\mathbf{x}, t, \frac{\mathbf{x}}{\varepsilon}\right)
\end{gathered}
$$


and

$$
\mathbf{A}^{\varepsilon} \mathbf{u}^{k}\left(\mathbf{x}, t, \frac{\mathbf{x}}{\varepsilon}\right)=\left(\varepsilon^{-2} \mathbf{A}^{1} \mathbf{u}^{k}+\varepsilon^{-1} \mathbf{A}^{2} \mathbf{u}^{k}+\mathbf{A}^{3} \mathbf{u}^{k}\right)\left(\mathbf{x}, t, \frac{\mathbf{x}}{\varepsilon}\right)
$$

where

$$
\begin{aligned}
\left(\mathbf{A}^{1} \mathbf{v}\right)_{j} & =-\frac{\partial}{\partial y_{\beta}}\left(a_{\alpha \beta}^{i j}(\mathbf{y}) \frac{\partial v_{i}}{\partial y_{\alpha}}\right), \\
\left(\mathbf{A}^{2} \mathbf{v}\right)_{j} & =-\frac{\partial}{\partial x_{\beta}}\left(a_{\alpha \beta}^{i j}(\mathbf{y}) \frac{\partial v_{i}}{\partial y_{\alpha}}\right)-\frac{\partial}{\partial y_{\beta}}\left(a_{\alpha \beta}^{i j}(\mathbf{y}) \frac{\partial v_{i}}{\partial x_{\alpha}}\right), \\
\left(\mathbf{A}^{3} \mathbf{v}\right)_{j} & =-\frac{\partial}{\partial x_{\beta}}\left(a_{\alpha \beta}^{i j}(\mathbf{y}) \frac{\partial v_{i}}{\partial x_{\alpha}}\right) .
\end{aligned}
$$

Hence substituting $(25)$ and $(26)$ into $\left(H P^{\varepsilon}\right)$ and identifying the terms with the same order of $\varepsilon$, we easily derive

$$
\begin{gathered}
\mathbf{A}^{1} \mathbf{u}^{0}=0, \\
\operatorname{div}_{\mathbf{y}} \mathbf{u}^{1}+\operatorname{div}_{\mathbf{x}} \mathbf{u}^{0}=g^{0}, \\
\mathbf{A}^{1} \mathbf{u}^{1}+\mathbf{A}^{2} \mathbf{u}^{0}+\nabla_{\mathbf{y}} p^{0}=0, \\
\partial_{t} \mathbf{u}^{0}+\mathbf{A}^{1} \mathbf{u}^{2}+\mathbf{A}^{2} \mathbf{u}^{1}+\mathbf{A}^{3} \mathbf{u}^{0}+\nabla_{\mathbf{y}} p^{1}+\nabla_{\mathbf{x}} p^{0}=\mathbf{f}^{0} .
\end{gathered}
$$

Using these equations, we will determine $\mathbf{u}_{0}, \mathbf{u}_{1}$ and $p_{0}$.

First, integrating (30) over $Y=[0,1]^{n}$, we eliminate $\mathbf{u}_{2}, p_{1}$ and obtain

$$
\partial_{t} \int_{Y} u_{j}^{0} d y-\frac{\partial}{\partial x_{\beta}} \int_{Y} a_{\alpha \beta}^{i j}(\mathbf{y})\left(\frac{\partial u_{i}^{1}}{\partial y_{\alpha}}+\frac{\partial u_{i}^{0}}{\partial x_{\alpha}}\right) d y+\frac{\partial}{\partial x_{j}} \int_{Y} p^{0} d y=f_{j}^{0}
$$

for each $j=1, \ldots, n$. Next, from (27), we deduce that $\mathbf{u}^{0}$ is a function of $(\mathbf{x}, t)$ only, i.e., $\mathbf{u}^{0}=\mathbf{u}^{0}(\mathbf{x}, t)$. In fact, if we multiply (27) by $\mathbf{u}^{0}$ and integrate by parts over $Y$, then by virtue of (18), we have

$$
\begin{aligned}
0 & =\int_{Y} a_{\alpha \beta}^{i j}(\mathbf{y}) \frac{\partial u_{i}^{0}}{\partial y_{\alpha}} \frac{\partial u_{j}^{0}}{\partial y_{\beta}} d y \\
& \geq \frac{\lambda}{4} \int_{Y}\left|\frac{\partial u_{i}^{0}}{\partial y_{\alpha}}+\frac{\partial u_{\alpha}^{0}}{\partial y_{i}}\right|^{2} d y=\frac{\lambda}{2} \int_{Y}\left(\left|\nabla_{\mathbf{y}} \mathbf{u}^{0}\right|^{2}+\left(\operatorname{div}_{\mathbf{y}} \mathbf{u}^{0}\right)^{2}\right) d y
\end{aligned}
$$

which implies that $\nabla_{\mathbf{y}} \mathbf{u}^{0} \equiv \mathbf{0}$. Moreover, since $\mathbf{u}^{1}$ is periodic in $\mathbf{y}$, it follows from (28) that

$$
\operatorname{div} \mathbf{u}^{0}=g^{0} .
$$

Then combining (28), (29) and (32), we deduce that for each $(\mathbf{x}, t) \in \Omega \times(0, T)$, $\left(\mathbf{u}^{1}, p^{0}\right)=\left(\mathbf{u}^{1}(\mathbf{x}, t, \cdot), p^{0}(\mathbf{x}, t, \cdot)\right)$ is a periodic solution of the Stokes equations

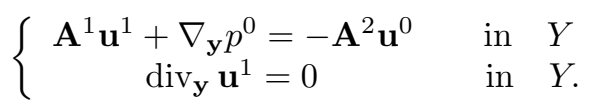

Associated with (33) is the bilinear form $a_{Y}$ on $\mathbf{H}_{p e r}^{1}(Y)$ defined by

$$
a_{Y}(\mathbf{v}, \mathbf{w})=\int_{Y} a_{\alpha \beta}^{i j}(\mathbf{y}) \frac{\partial v_{i}}{\partial y_{\alpha}} \frac{\partial w^{j}}{\partial y_{\beta}} d y \quad \text { for } \quad \mathbf{v}, \mathbf{w} \in \mathbf{H}_{p e r}^{1}(Y),
$$


where $\mathbf{H}_{\text {per }}^{1}(Y)$ is the closure of the space of all smooth periodic vector fields in $\mathbb{R}^{n}$ with respect to the $\mathbf{H}^{1}(Y)$-norm. $L_{\text {per }}^{2}(Y)$ and $\mathbf{L}_{\text {per }}^{2}(Y)$ are defined similarly.

Lemma 2. For each $\mathbf{F} \in\left[L_{p e r}^{2}(Y)\right]^{n^{2}}$, there exists a unique weak solution $(\mathbf{v}, q)$ in $\mathbf{H}_{\text {per }}^{1}(Y) \times L_{\text {per }}^{2}(Y)$ to the problem

$$
\left\{\begin{array}{c}
\mathbf{A}^{1} \mathbf{v}+\nabla q=\operatorname{div} \mathbf{F} \quad \text { in } \quad Y, \\
\operatorname{div} \mathbf{v}=0 \quad \text { in } Y, \\
\int_{Y} \mathbf{v} d y=0, \quad \int_{Y} q d y=0 .
\end{array}\right.
$$

Proof. By virtue of (4) and (18), we easily deduce that $a_{Y}$ is a continuous coercive bilinear form on $\mathbf{H}_{\text {per }}^{1}(Y) / \mathbb{R}=\left\{\mathbf{v} \in \mathbf{H}_{\text {per }}^{1}(Y): \int_{Y} \mathbf{v} d y=0\right\}$. Moreover, the mapping

$$
\mathbf{w} \mapsto-\left(\mathbf{F}, \nabla_{\mathbf{y}} \mathbf{w}\right)_{\mathbf{L}^{2}(Y)}=-\int_{Y} \mathbf{F} \cdot \nabla_{\mathbf{y}} \mathbf{w} d y
$$

is a continuous linear functional on $\mathbf{H}_{\text {per }}^{1}(Y) / \mathbb{R}$. Hence the Lax-Milgram theorem allows us to deduce the existence of a unique vector field

$$
\mathbf{v} \in \mathbf{H}_{p e r, \sigma}^{1}(Y) / \mathbb{R}=\left\{\mathbf{v} \in \mathbf{H}_{\text {per }}^{1}(Y) / \mathbb{R}: \operatorname{div} \mathbf{v}=0\right\}
$$

such that

$$
a_{Y}(\mathbf{v}, \mathbf{w})=-\left(\mathbf{F}, \nabla_{\mathbf{y}} \mathbf{w}\right)_{\mathbf{L}^{2}(Y)} \quad \text { for all } \quad \mathbf{w} \in \mathbf{H}_{p e r, \sigma}^{1}(Y) / \mathbb{R} .
$$

Then it is quite standard to prove the existence of a unique $q \in L_{p e r}^{2}(Y) / \mathbb{R}$ such that

$$
a_{Y}(\mathbf{v}, \mathbf{w})-(q, \operatorname{div} \mathbf{w})_{L^{2}(Y)}=-\left(\mathbf{F}, \nabla_{\mathbf{y}} \mathbf{w}\right)_{\mathbf{L}^{2}(Y)}
$$

for all $\mathbf{w} \in \mathbf{H}_{\text {per }}^{1}(Y)$. This completes the proof of Lemma 2 .

Since $\mathbf{u}^{0}=\mathbf{u}^{0}(\mathbf{x}, t)$ is independent of $\mathbf{y}$, we can find general periodic solutions of (33) by applying Lemma 2. To do this, we observe that

$$
\begin{aligned}
-\left(\mathbf{A}^{2} \mathbf{u}^{0}\right)_{j} & =\frac{\partial}{\partial y_{\beta}}\left(a_{\alpha \beta}^{i j}(\mathbf{y}) \frac{\partial u_{i}^{0}}{\partial x_{\alpha}}\right)=\frac{\partial}{\partial y_{\beta}}\left(a_{\alpha \beta}^{i j}(\mathbf{y})\right) \frac{\partial u_{i}^{0}}{\partial x_{\alpha}} \\
& =\frac{\partial}{\partial y_{\beta}}\left(a_{\gamma \beta}^{k j}(\mathbf{y}) \frac{\partial}{\partial y_{\alpha}}\left(y_{\gamma} \delta_{k i}\right)\right) \frac{\partial u_{k}^{0}}{\partial x_{\gamma}}=-\left(\mathbf{A}^{1}\left(y_{\gamma} \mathbf{e}^{k}\right) \frac{\partial u_{k}^{0}}{\partial x_{\gamma}}\right)_{j}
\end{aligned}
$$

for each $j$, that is,

$$
-\mathbf{A}^{2} \mathbf{u}^{0}=-\mathbf{A}^{1}\left(y_{\gamma} \mathbf{e}^{k}\right) \frac{\partial u_{k}^{0}}{\partial x_{\gamma}} .
$$

Recall that $\left\{\mathbf{e}^{k}\right\}_{1 \leq k \leq n}$ is the canonical basis of $\mathbb{R}^{n}$ and $e_{i}^{k}=\delta_{k i}$. In view of Lemma 2, we may define the compensating functions $\chi_{\alpha}^{i}$ and $q_{\alpha}^{i}$ as follows.

Definition 3. For each $\alpha, i$ with $1 \leq \alpha, i \leq n$, let $\left(\chi_{\alpha}^{i}, q_{\alpha}^{i}\right) \in \mathbf{H}_{p e r}^{1}(Y) \times L_{p e r}^{2}(Y)$ be the unique weak solution to the problem (35) with $\operatorname{div} \mathbf{F}=\mathbf{A}^{1}\left(y_{\alpha} \mathbf{e}^{i}\right)$. In particular, we have

$$
a_{Y}\left(\chi_{\alpha}^{i}, \mathbf{v}\right)-\left(q_{\alpha}^{i}, \operatorname{div} \mathbf{v}\right)_{L^{2}(Y)}=a_{Y}\left(y_{\alpha} \mathbf{e}^{i}, \mathbf{v}\right) \quad \text { for all } \quad \mathbf{v} \in \mathbf{H}_{p e r}^{1}(Y) .
$$


Then by virtue of (33) and (36), we deduce that

$$
\mathbf{u}^{1}=-\chi_{\alpha}^{i}(\mathbf{y}) \frac{\partial u_{i}^{0}}{\partial x_{\alpha}}+\tilde{\mathbf{u}}^{1} \quad \text { and } \quad p^{0}=-q_{\alpha}^{i}(\mathbf{y}) \frac{\partial u_{i}^{0}}{\partial x_{\alpha}}+\tilde{p}^{0}
$$

for some functions $\tilde{\mathbf{u}}^{1}(\mathbf{x}, t)$ and $\tilde{p}^{0}(\mathbf{x}, t)$. Substituting (37) into (31), we finally derive

$$
\partial_{t} u_{j}^{0}-\frac{\partial}{\partial x_{\beta}}\left(\tilde{a}_{\alpha \beta}^{i j} \frac{\partial u_{i}^{0}}{\partial x_{\alpha}}\right)+\frac{\partial}{\partial x_{j}} \bar{p}^{0}=f_{j}^{0}
$$

for each $j=1, \ldots, n$, where

$$
\bar{p}^{0}=\bar{p}^{0}(\mathbf{x}, t)=\int_{Y} p^{0}(\mathbf{x}, t, \mathbf{y}) d y
$$

and

$$
\begin{aligned}
\tilde{a}_{\alpha \beta}^{i j} & =\int_{Y}\left[a_{\alpha \beta}^{i j}(\mathbf{y})-a_{\gamma \beta}^{k j}(\mathbf{y}) \frac{\partial}{\partial y_{\gamma}}\left(\chi_{\alpha}^{i}\right)_{k}(\mathbf{y})\right] d y \\
& =\int_{Y} a_{\gamma \delta}^{k l}(\mathbf{y}) \frac{\partial}{\partial y_{\gamma}}\left(y_{\alpha} e_{k}^{i}-\left(\chi_{\alpha}^{i}\right)_{k}(\mathbf{y})\right) \frac{\partial}{\partial y_{\delta}}\left(y_{\beta} e_{l}^{j}\right) d y .
\end{aligned}
$$

We can rewrite the constants $\tilde{a}_{\alpha \beta}^{i j}$ in another useful forms. Observe from the definition of $\chi_{\alpha}^{i}$ that

$$
a_{Y}\left(\chi_{\alpha}^{i}, \mathbf{w}\right)=a_{Y}\left(y_{\alpha} \mathbf{e}^{i}, \mathbf{w}\right)
$$

for all $\mathbf{w} \in \mathbf{H}_{\text {per }}^{1}(Y)$ with $\operatorname{div} \mathbf{w}=0$. Taking $\mathbf{w}=\chi_{\beta}^{j}$ in $(40)$, we deduce from (34) and (39) that

$$
\tilde{a}_{\alpha \beta}^{i j}=a_{Y}\left(y_{\alpha} \mathbf{e}^{i}-\chi_{\alpha}^{i}, y_{\beta} \mathbf{e}^{j}\right)=a_{Y}\left(y_{\alpha} \mathbf{e}^{i}-\chi_{\alpha}^{i}, y_{\beta} \mathbf{e}^{j}-\chi_{\beta}^{j}\right) .
$$

\section{Lemma 4.}

and

$$
\tilde{a}_{\alpha \beta}^{i j}=\tilde{a}_{i \beta}^{\alpha j}=\tilde{a}_{\alpha j}^{i \beta}
$$

$$
\tilde{a}_{\alpha \beta}^{i j} \xi_{\alpha}^{i} \xi_{\beta}^{j} \geq \tilde{\lambda}|\xi|^{2} \quad \text { for all } \quad \xi \in \mathcal{S}(n)
$$

Here $\mathcal{S}(n)$ denotes the space of all $n \times n$-real symmetric matrices and $\tilde{\lambda}$ is a positive constant.

Proof. Since $a_{\gamma \delta}^{k l}(\mathbf{y})=a_{\gamma l}^{k \delta}(\mathbf{y})$, it follows that $a_{Y}\left(\mathbf{w}, y_{\beta} \mathbf{e}^{j}\right)=a_{Y}\left(\mathbf{w}, y_{j} \mathbf{e}^{\beta}\right)$ for all $\mathbf{w} \in \mathbf{H}^{1}(Y)$. Taking $\mathbf{w}=y_{\alpha} \mathbf{e}^{i}-\chi_{\alpha}^{i}$, we deduce that $\tilde{a}_{\alpha \beta}^{i j}=\tilde{a}_{\alpha j}^{i \beta}$. Similarly, since $a_{Y}\left(y_{\alpha} \mathbf{e}^{i}, \mathbf{w}\right)=a_{Y}\left(y_{i} \mathbf{e}^{\alpha}, \mathbf{w}\right)$ for all $\mathbf{w} \in \mathbf{H}^{1}(Y)$, it follows from (40) that

$$
a_{Y}\left(\chi_{\alpha}^{i}, \mathbf{w}\right)=a_{Y}\left(y_{\alpha} \mathbf{e}^{i}, \mathbf{w}\right)=a_{Y}\left(y_{i} \mathbf{e}^{\alpha}, \mathbf{w}\right)=a_{Y}\left(\chi_{i}^{\alpha}, \mathbf{w}\right)
$$

for all $\mathbf{w} \in \mathbf{H}_{\text {per }}^{1}(Y)$ with $\operatorname{div} \mathbf{w}=0$. Taking $\mathbf{w}=\chi_{\alpha}^{i}-\chi_{i}^{\alpha}$, we deduce that $\chi_{\alpha}^{i}=\chi_{i}^{\alpha}$ because $\int_{Y} \chi_{\alpha}^{i} d y=\int_{Y} \chi_{i}^{\alpha} d y=0$ by Definition 3 . Hence by virtue of (41), we have

$$
\begin{aligned}
\tilde{a}_{\alpha \beta}^{i j} & =a_{Y}\left(y_{\alpha} \mathbf{e}^{i}-\chi_{\alpha}^{i}, y_{\beta} \mathbf{e}^{j}\right)=a_{Y}\left(y_{\alpha} \mathbf{e}^{i}, y_{\beta} \mathbf{e}^{j}\right)-a_{Y}\left(\chi_{\alpha}^{i}, y_{\beta} \mathbf{e}^{j}\right) \\
& =a_{Y}\left(y_{i} \mathbf{e}^{\alpha}, y_{\beta} \mathbf{e}^{j}\right)-a_{Y}\left(\chi_{i}^{\alpha}, y_{\beta} \mathbf{e}^{j}\right)=a_{Y}\left(y_{i} \mathbf{e}^{\alpha}-\chi_{i}^{\alpha}, y_{\beta} \mathbf{e}^{j}\right)=\tilde{a}_{i \beta}^{\alpha j} .
\end{aligned}
$$


This proves the symmetry of $\tilde{a}_{\alpha \beta}^{i j}$. To prove the ellipticity, let us suppose that $\tilde{a}_{\alpha \beta}^{i j} \xi_{\alpha}^{i} \xi_{\beta}^{j} \leq 0$ for some $\xi \in \mathcal{S}(n)$. Then setting $\mathbf{w}=\xi_{\alpha}^{i}\left(y_{\alpha} \mathbf{e}^{i}-\chi_{\alpha}^{i}(y)\right)$, we have

and so

$$
0 \geq \tilde{a}_{\alpha \beta}^{i j} \xi_{\alpha}^{i} \xi_{\beta}^{j}=a_{Y}(\mathbf{w}, \mathbf{w}) \geq \frac{\lambda}{4} \int_{Y}\left|\frac{\partial w_{k}}{\partial y_{\gamma}}+\frac{\partial w_{\gamma}}{\partial y_{k}}\right|^{2} d y
$$

$$
\frac{\partial w_{k}}{\partial y_{\gamma}}+\frac{\partial w_{\gamma}}{\partial y_{k}}=0 \quad \text { for } \quad 1 \leq k, \gamma \leq n
$$

Hence it follows from a classical result in mechanics (see [11] for instance) that there is an anti-symmetric matrix $\eta=\left(\eta_{\alpha}^{i}\right)$ and a vector $\mathbf{b}$ such that $\mathbf{w}(\mathbf{y})=\eta \cdot \mathbf{y}+\mathbf{b}$ for all $\mathbf{y} \in Y$. Noting that $(\eta \cdot \mathbf{y})_{k}=\eta_{\alpha}^{k} y_{\alpha}=\eta_{\alpha}^{i} y_{\alpha} e_{k}^{i}$ for each $k$, we deduce that

$$
\xi_{\alpha}^{i}\left(y_{\alpha} \mathbf{e}^{i}-\chi_{\alpha}^{i}(\mathbf{y})\right)=\eta_{\alpha}^{i} y_{\alpha} \mathbf{e}^{i}+\mathbf{b} \quad \text { or } \quad\left(\xi_{\alpha}^{i}-\eta_{\alpha}^{i}\right) y_{\alpha} \mathbf{e}^{i}=\xi_{\alpha}^{i} \chi_{\alpha}^{i}(\mathbf{y})+\mathbf{b} .
$$

Hence the periodicity of $\chi_{\alpha}^{i}$ allows us to conclude that

$$
\xi-\eta=0 \quad \text { and so } \quad \xi=\eta=0 .
$$

We have shown that $\tilde{a}_{\alpha \beta}^{i j} \xi_{\alpha}^{i} \xi_{\beta}^{j}>0$ for all $\xi \in \mathcal{S}(n) \backslash\{0\}$, which implies the ellipticity of $\tilde{a}_{\alpha \beta}^{i j}$. The proof of Lemma 4 is completed.

Remark 5. Assume that $a_{\gamma \delta}^{k l}(\mathbf{y})=a_{\delta \gamma}^{l k}(\mathbf{y})$ for a.e. $\mathbf{y} \in \mathbb{R}^{n}$ and all $\gamma, \delta, k, l$. Then it follows immediately from (34) and (41) that $\tilde{a}_{\alpha \beta}^{i j}=\tilde{a}_{\beta \alpha}^{j i}$ for all $\alpha, \beta, i, j$.

In view of Lemma 4, we can adapt the proof of Theorem 1 to obtain the corresponding well-posedness result for the homogenized problem:

$$
\left\{\begin{aligned}
\partial_{t} \mathbf{u}^{0}+\mathbf{A}^{0} \mathbf{u}^{0}+\nabla p^{0}=\mathbf{f}^{0} & \text { in } \quad \Omega \times(0, T) \\
\operatorname{div} \mathbf{u}^{0}=g^{0} & \text { in } \Omega \times(0, T) \\
\mathbf{u}^{0}=\mathbf{u}_{b}^{0} & \text { on } \quad \Omega \times(0, T) \\
\mathbf{u}^{0}=\mathbf{u}_{0} & \text { on } \Omega \times\{0\},
\end{aligned}\right.
$$

where the homogenized operator $\mathbf{A}^{0}$ is given by

$$
\left(\mathbf{A}^{0} \mathbf{v}\right)_{j}=-\frac{\partial}{\partial x_{\beta}}\left(\tilde{a}_{\alpha \beta}^{i j} \frac{\partial v_{i}}{\partial x_{\alpha}}\right) \quad(j=1, \ldots, n) .
$$

Theorem 6. Let the data $\mathbf{f}^{0}, g^{0}, \mathbf{u}_{b}^{0}$ and $\mathbf{u}_{0}^{0}$ satisfy the following conditions:

$$
\begin{gathered}
\mathbf{f}^{0} \in L^{2}\left(0, T ; \mathbf{H}^{-1}(\Omega)\right), \quad \operatorname{div} \mathbf{G}^{0}=g^{0} \quad \text { in } \quad \Omega \times(0, T), \\
\mathbf{G}^{0}=\mathbf{u}_{b}^{0} \quad \text { on } \quad \partial \Omega \times(0, T) \quad \text { and } \quad \mathbf{u}_{0}^{0}-\mathbf{G}^{0}(0) \in H
\end{gathered}
$$

for some $\mathbf{G}^{0} \in \mathbf{W}_{T}$. Then there exists a unique weak solution $\mathbf{u}^{0}$ to the homogenized problem $\left(H P^{0}\right)$ in the sense that

$$
\mathbf{u}^{0}-\mathbf{G}^{0} \in \mathbf{V}_{T}
$$

and

$$
\left(\mathbf{u}^{0}(t)-\mathbf{u}_{0}^{0}, \mathbf{w}\right)_{\mathbf{L}^{2}(\Omega)}+\int_{0}^{t} a^{0}\left(\mathbf{u}^{0}(\tau), \mathbf{w}\right) d \tau=\int_{0}^{t}\left\langle\mathbf{f}^{0}(\tau), \mathbf{w}\right\rangle d \tau
$$


for all $t \in[0, T]$ and $\mathbf{w} \in V$. Here the bilinear form $a^{0}$ on $\mathbf{H}^{1}(\Omega)$ is given by

$$
a^{0}(\mathbf{v}, \mathbf{w})=\int_{\Omega} \tilde{a}_{\alpha \beta}^{i j} \frac{\partial v_{i}}{\partial x_{\alpha}} \frac{\partial w_{j}}{\partial x_{\beta}} d x \quad \text { for } \quad \mathbf{v}, \mathbf{w} \in \mathbf{H}^{1}(\Omega) .
$$

\section{The homogenization theorem}

We are now ready to prove the following homogenization theorem.

Theorem 7. For each $\varepsilon>0$, let $\mathbf{u}^{\varepsilon}$ be the unique weak solution to the problem $\left(H P^{\varepsilon}\right)$ with the data $\mathbf{f}^{\varepsilon}, g^{\varepsilon}, \mathbf{u}_{b}^{\varepsilon}$ and $\mathbf{u}_{0}^{\varepsilon}$ satisfying the conditions (14), (15) and (16). Assume further that

$$
\left\{\begin{aligned}
\mathbf{f}^{\varepsilon} & \rightarrow \mathbf{f}^{0} & \text { strongly in } & L^{2}\left(0, T ; \mathbf{H}^{-1}(\Omega)\right) \\
g^{\varepsilon} & \rightarrow g^{0} & \text { strongly in } & L^{2}\left(0, T ; L^{2}(\Omega)\right) \\
\mathbf{G}^{\varepsilon} & \rightarrow \mathbf{G}^{0} & \text { weakly in } & \mathbf{W}_{T} \\
\mathbf{u}_{0}^{\varepsilon} & \rightarrow \mathbf{u}_{0}^{0} & \text { weakly in } & \mathbf{L}^{2}(\Omega) .
\end{aligned}\right.
$$

Then we have

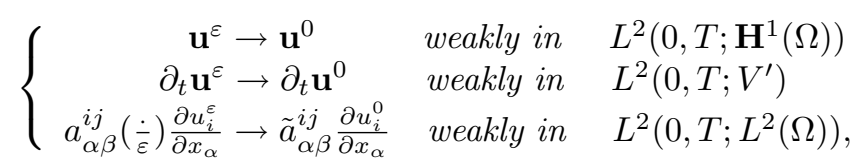

where $\mathbf{u}^{0}$ is the unique weak solution to the homogenized problem $\left(H P^{0}\right)$ with the data $\mathbf{f}^{0}, g^{0}=\operatorname{div} \mathbf{G}^{0}, \mathbf{u}_{b}^{0}=\left.\mathbf{G}^{0}\right|_{\partial \Omega \times(0, T)}$ and $\mathbf{u}_{0}^{0}$.

Remark 8. From (46), we can also deduce that

$$
\mathbf{u}^{\varepsilon} \rightarrow \mathbf{u}^{0} \quad \text { strongly in } \quad L^{2}\left(0, T ; \mathbf{L}^{2}(\Omega)\right)
$$

and

$$
\left(\mathbf{u}^{\varepsilon}, \mathbf{w}\right)_{\mathbf{L}^{2}(\Omega)} \rightarrow\left(\mathbf{u}^{0}, \mathbf{w}\right)_{\mathbf{L}^{2}(\Omega)} \quad \text { uniformly on } \quad[0, T]
$$

for all $\mathbf{w} \in \mathbf{L}^{2}(\Omega)$.

Proof of Remark 8. Let us denote

$$
\mathbf{v}^{\varepsilon}=\mathbf{u}^{\varepsilon}-\mathbf{G}^{\varepsilon} \text { and } \quad \mathbf{v}^{0}=\mathbf{u}^{0}-\mathbf{G}^{0} .
$$

Then since $\mathbf{G}^{\varepsilon} \rightarrow \mathbf{G}^{0}$ weakly in $\mathbf{W}_{T}$, it follows from (46) that $\mathbf{v}^{\varepsilon} \rightarrow \mathbf{v}^{0}$ weakly in $\mathbf{V}_{T}$. Hence using the well-known compactness result $\mathbf{V}_{T} \hookrightarrow \hookrightarrow L^{2}(0, T ; H)$, we first deduce that

$$
\mathbf{v}^{\varepsilon} \rightarrow \mathbf{v}^{0} \quad \text { strongly in } \quad L^{2}\left(0, T ; \mathbf{L}^{2}(\Omega)\right) .
$$

Next, we will show that

$$
\left(\mathbf{v}^{\varepsilon}, \mathbf{w}\right)_{\mathbf{L}^{2}(\Omega)} \rightarrow\left(\mathbf{v}^{0}, \mathbf{w}\right)_{\mathbf{L}^{2}(\Omega)} \quad \text { uniformly on } \quad[0, T]
$$

for all $\mathbf{w} \in \mathbf{L}^{2}(\Omega)$. It suffices to show that if $\mathbf{w} \in \mathbf{L}^{2}(\Omega)$ and $\varepsilon_{m} \rightarrow 0$ as $m \rightarrow \infty$, then the sequence $\left\{\left(\mathbf{v}^{\varepsilon_{m}}, \mathbf{w}\right)_{\mathbf{L}^{2}(\Omega)}\right\}$ has a subsequence converging to $\left(\mathbf{v}^{0}, \mathbf{w}\right)_{\mathbf{L}^{2}(\Omega)}$ uniformly on $[0, T]$. This is proved first for each $\mathbf{w} \in$ $V$. Suppose that $\varepsilon_{m} \rightarrow 0$ as $m \rightarrow \infty$. Then it follows from (49) that 
$\int_{0}^{T}\left\|\mathbf{v}^{\varepsilon_{m}}(t)-\mathbf{v}^{0}(t)\right\|_{\mathbf{L}^{2}(\Omega)}^{2} d t \rightarrow 0$ as $m \rightarrow \infty$. Hence there exists a subsequence of $\left\{\varepsilon_{m}\right\}$, still denoted by $\left\{\varepsilon_{m}\right\}$, such that $\mathbf{v}^{\varepsilon_{m}}\left(t_{0}\right) \rightarrow \mathbf{v}^{0}\left(t_{0}\right)$ strongly in $\mathbf{L}^{2}(\Omega)$ for at least one $t_{0} \in[0, T]$. Moreover, since $\partial_{t} \mathbf{v}^{\varepsilon} \rightarrow \partial_{t} \mathbf{v}^{0}$ weakly in $L^{2}\left(0, T ; V^{\prime}\right)$, we have

$$
\begin{aligned}
\left(\mathbf{v}^{\varepsilon_{m}}(t), \mathbf{w}\right)_{\mathbf{L}^{2}(\Omega)} & =\left(\mathbf{v}^{\varepsilon_{m}}\left(t_{0}\right), \mathbf{w}\right)_{\mathbf{L}^{2}(\Omega)}+\int_{t_{0}}^{t}\left\langle\partial_{t} \mathbf{v}^{\varepsilon_{m}}(\tau), \mathbf{w}\right\rangle d \tau \\
& \rightarrow\left(\mathbf{v}^{0}\left(t_{0}\right), \mathbf{w}\right)_{\mathbf{L}^{2}(\Omega)}+\int_{t_{0}}^{t}\left\langle\partial_{t} \mathbf{v}^{0}(\tau), \mathbf{w}\right\rangle d \tau \\
& =\left(\mathbf{v}^{0}(t), \mathbf{w}\right)_{\mathbf{L}^{2}(\Omega)} \quad \text { for all } \quad t \in[0, T],
\end{aligned}
$$

which proves the pointwise convergence of $\left\{\left(\mathbf{v}^{\varepsilon_{m}}, \mathbf{w}\right)_{\mathbf{L}^{2}(\Omega)}\right\}$ on $[0, T]$ as $\varepsilon \rightarrow 0$. The uniform convergence now follows from the Ascoli-Arzelà theorem because even the whole family $\left\{\left(\mathbf{v}^{\varepsilon}, \mathbf{w}\right)_{\mathbf{L}^{2}(\Omega)}\right\}_{\varepsilon>0}$ is equicontinuous on $[0, T]$. In fact, if $0 \leq t<t+h \leq T$, then

$$
\begin{aligned}
\left|\left(\mathbf{v}^{\varepsilon}(t+h)-\mathbf{v}^{\varepsilon}(t), \mathbf{w}\right)_{\mathbf{L}^{2}(\Omega)}\right| & =\left|\int_{t}^{t+h}\left\langle\partial_{t} \mathbf{v}^{\varepsilon}(\tau), \mathbf{w}\right\rangle d \tau\right| \\
& \leq\left(\int_{t}^{t+h}\left\|\partial_{t} \mathbf{v}^{\varepsilon}(\tau)\right\|_{V^{\prime}} d \tau\right)\|\mathbf{w}\|_{V} \leq C h^{\frac{1}{2}}
\end{aligned}
$$

for some constant $C$ independent of $t$ and $\varepsilon$. This proves (50) for all $\mathbf{v} \in V$. A simple density argument allows us to deduce (50) for all $\mathbf{v} \in H$. Finally, using the Helmholtz projection of $\mathbf{L}^{2}(\Omega)$ onto $H$, we easily deduce (50) for all $\mathbf{v} \in \mathbf{L}^{2}(\Omega)$ because $\mathbf{v}^{\varepsilon}(t), \mathbf{v}^{0}(t) \in H$ for all $t \in[0, T]$.

Using the embedding result $\mathbf{W}_{T} \hookrightarrow \hookrightarrow L^{2}\left(0, T ; \mathbf{L}^{2}(\Omega)\right)$, we can also adapt the above argument to show that

$$
\mathbf{G}^{\varepsilon} \rightarrow \mathbf{G}^{0} \quad \text { strongly in } \quad L^{2}\left(0, T ; \mathbf{L}^{2}(\Omega)\right)
$$

and

$$
\left(\mathbf{G}^{\varepsilon}, \mathbf{w}\right)_{\mathbf{L}^{2}(\Omega)} \rightarrow\left(\mathbf{G}^{0}, \mathbf{w}\right)_{\mathbf{L}^{2}(\Omega)} \quad \text { uniformly on }[0, T]
$$

for all $\mathbf{w} \in \mathbf{L}^{2}(\Omega)$. This completes the proofs of (47) and (48).

Proof of Theorem 7 . We have only to show that for each sequence $\left\{\varepsilon_{m}\right\}$ with $\varepsilon_{m} \rightarrow 0,\left\{\mathbf{u}^{\varepsilon_{m}}\right\}$ has a subsequence converging to a (unique by Theorem 6 ) weak solution to the homogenized problem $\left(H P^{0}\right)$. Let us denote

$$
{ }^{\varepsilon_{m}} \xi_{\beta}^{j}={ }^{\varepsilon_{m}} \xi_{\beta}^{j}(\mathbf{x}, t)=a_{\alpha \beta}^{i j}\left(\frac{\mathbf{x}}{\varepsilon_{m}}\right) \frac{\partial u_{i}^{\varepsilon_{m}}}{\partial x_{\alpha}}(\mathbf{x}, t) .
$$

Then by virtue of Theorem 1 , the sequences $\left\{\mathbf{u}^{\varepsilon_{m}}\right\}$ and $\left\{\varepsilon_{m} \xi_{\beta}^{j}\right\}$ are $\varepsilon_{m}$-uniformly bounded in $\mathbf{W}_{T}+\mathbf{V}_{T}$ and $L^{2}\left(0, T ; L^{2}(\Omega)\right)$, respectively. Hence standard compactness results enable us to extract subsequences, still denoted by $\left\{\mathbf{u}^{\varepsilon_{m}}\right\}$ 
and $\left\{\varepsilon_{m} \xi_{\beta}^{j}\right\}$, which converge to $\mathbf{u} \in \mathbf{W}_{T}+\mathbf{V}_{T}$ and $\xi_{\beta}^{j} \in L^{2}\left(0, T ; L^{2}(\Omega)\right)$, respectively, in the following sense:

$$
\left\{\begin{array}{rlrl}
\mathbf{u}^{\varepsilon_{m}} & \rightarrow \mathbf{u} & \text { weakly in } & L^{2}\left(0, T ; \mathbf{H}^{1}(\Omega)\right) \\
\partial_{t} \mathbf{u}^{\varepsilon_{m}} & \rightarrow \partial_{t} \mathbf{u} & \text { weakly in } & L^{2}\left(0, T ; V^{\prime}\right) \\
\mathbf{u}^{\varepsilon_{m}} & \rightarrow \mathbf{u} & \text { strongly in } & L^{2}\left(0, T ; \mathbf{L}^{2}(\Omega)\right) \\
\varepsilon_{m} \xi_{\beta}^{j} & \rightarrow \xi_{\beta}^{j} \quad \text { weakly in } & L^{2}\left(0, T ; L^{2}(\Omega)\right)
\end{array}\right.
$$

and

$$
\left(\mathbf{u}^{\varepsilon_{m}}, \mathbf{w}\right)_{\mathbf{L}^{2}(\Omega)} \rightarrow(\mathbf{u}, \mathbf{w})_{\mathbf{L}^{2}(\Omega)} \quad \text { uniformly on } \quad[0, T]
$$

for all $\mathbf{w} \in \mathbf{L}^{2}(\Omega)$. In view of (51) and (52), we easily deduce from (23) and (24) as $\varepsilon_{m} \rightarrow 0$ that

$$
\mathbf{u}-\mathbf{G}^{0} \in \mathbf{V}_{T}
$$

and

$$
\int_{\Omega}\left(\mathbf{u}(t)-\mathbf{u}_{0}\right) \cdot \mathbf{w} d x+\int_{0}^{t} \int_{\Omega} \xi_{\beta}^{j} \frac{\partial w_{j}}{\partial x_{\beta}} d x d \tau=\int_{0}^{t}\langle\mathbf{f}(\tau), \mathbf{w}\rangle d \tau
$$

for all $t \in[0, T]$ and $\mathbf{w} \in V$. Hence to prove the theorem, it remains to show that

$$
\xi_{\beta}^{j}=\tilde{a}_{\alpha \beta}^{i j} \frac{\partial u_{i}}{\partial x_{\alpha}} \quad \text { for each } \quad 1 \leq j, \beta \leq n,
$$

which implies, combined with (53) and (54), that $\mathbf{u}$ is a weak solution to the homogenized problem $\left(H P^{0}\right)$.

Our proof of (55) relies on Tartar's classical method of oscillating test functions; see [2] and [5] for convenient references. To apply this method, we first reduce $(24)$ to an equivalent weak formulation with general test functions in $\mathbf{H}_{0}^{1}(\Omega)$ allowed. Let $t \in[0, T]$ be fixed. Then the validity of (24) for all $v \in V$ implies that the mapping

$$
\mathbf{w} \mapsto\left(\mathbf{u}^{\varepsilon}(t)-\mathbf{u}_{0}^{\varepsilon}, \mathbf{w}\right)_{\mathbf{L}^{2}(\Omega)}+\int_{0}^{t} a^{\varepsilon}\left(\mathbf{u}^{\varepsilon}(\tau), \mathbf{w}\right) d \tau-\int_{0}^{t}\left\langle\mathbf{f}^{\varepsilon}(\tau), \mathbf{w}\right\rangle d \tau
$$

is a bounded linear functional on $\mathbf{H}_{0}^{1}(\Omega)$ vanishing identically on $V$. Hence it follows from a standard result in fluid mechanics (see [7] or [13]) that there exists a unique scalar field $P^{\varepsilon}(t) \in L^{2}(\Omega) / \mathbb{R}$ such that

$$
\begin{aligned}
& \left(\mathbf{u}^{\varepsilon}(t)-\mathbf{u}_{0}^{\varepsilon}, \mathbf{w}\right)_{\mathbf{L}^{2}(\Omega)}+\int_{0}^{t} a^{\varepsilon}\left(\mathbf{u}^{\varepsilon}(\tau), \mathbf{w}\right) d \tau-\left(P^{\varepsilon}(t), \operatorname{div} \mathbf{w}\right)_{L^{2}(\Omega)} \\
= & \int_{0}^{t}\left\langle\mathbf{f}^{\varepsilon}(\tau), \mathbf{w}\right\rangle d \tau
\end{aligned}
$$

for all $\mathbf{w} \in \mathbf{H}_{0}^{1}(\Omega)$. Moreover, it can be shown that $P^{\varepsilon} \in C\left([0, T] ; L^{2}(\Omega)\right)$ and

$$
\left\|P^{\varepsilon}\right\|_{C\left([0, T] ; L^{2}(\Omega)\right)} \leq C\left(\left\|\mathbf{u}_{0}^{\varepsilon}\right\|_{\mathbf{L}^{2}(\Omega)}^{2}+\left\|\mathbf{G}^{\varepsilon}\right\|_{\mathbf{W}_{T}}^{2}+\left\|\mathbf{f}^{\varepsilon}\right\|_{L^{2}\left(0, T ; \mathbf{H}^{-1}(\Omega)\right)}^{2}\right) .
$$


To show this, let $0 \leq s \leq t \leq T$ be fixed. Then from (56), we derive (58)

$$
\begin{aligned}
& \left(\mathbf{u}^{\varepsilon}(t)-\mathbf{u}^{\varepsilon}(s), \mathbf{w}\right)_{\mathbf{L}^{2}(\Omega)}+\int_{s}^{t} a^{\varepsilon}\left(\mathbf{u}^{\varepsilon}(\tau), \mathbf{w}\right) d \tau-\left(P^{\varepsilon}(t)-P^{\varepsilon}(s), \operatorname{div} \mathbf{w}\right)_{L^{2}(\Omega)} \\
= & \int_{s}^{t}\left\langle\mathbf{f}^{\varepsilon}(\tau), \mathbf{w}\right\rangle d \tau .
\end{aligned}
$$

On the other hand, by virtue of a classical result due to Bogovskii (see [3] and $[7])$, there exists a vector field $\mathbf{w}^{\varepsilon}(s, t) \in \mathbf{H}_{0}^{1}(\Omega)$ such that

$\operatorname{div} \mathbf{w}^{\varepsilon}(s, t)=P^{\varepsilon}(t)-P^{\varepsilon}(s)$ and $\left\|\mathbf{w}^{\varepsilon}(s, t)\right\|_{\mathbf{H}^{1}(\Omega)} \leq C\left\|P^{\varepsilon}(t)-P^{\varepsilon}(s)\right\|_{L^{2}(\Omega)}$.

Hence from (58), we deduce that

$$
\begin{aligned}
& \left\|P^{\varepsilon}(t)-P^{\varepsilon}(s)\right\|_{L^{2}(\Omega)}^{2} \\
\leq & C\left\|\mathbf{u}^{\varepsilon}(t)-\mathbf{u}^{\varepsilon}(s)\right\|_{\mathbf{L}^{2}(\Omega)}\left\|\mathbf{w}^{\varepsilon}(s, t)\right\|_{\mathbf{H}^{1}(\Omega)} \\
& +C\left(\int_{s}^{t}\left\|\nabla \mathbf{u}^{\varepsilon}(\tau)\right\|_{\mathbf{L}^{2}(\Omega)} d \tau+\int_{s}^{t}\left\|\mathbf{f}^{\varepsilon}(\tau)\right\|_{\mathbf{H}^{-1}(\Omega)} d \tau\right)\left\|\mathbf{w}^{\varepsilon}(s, t)\right\|_{\mathbf{H}^{1}(\Omega)}
\end{aligned}
$$

and so

$$
\begin{aligned}
& \left\|P^{\varepsilon}(t)-P^{\varepsilon}(s)\right\|_{L^{2}(\Omega)} \\
\leq & C\left(\left\|\mathbf{u}^{\varepsilon}(t)-\mathbf{u}^{\varepsilon}(s)\right\|_{\mathbf{L}^{2}(\Omega)}+\int_{s}^{t}\left\|\nabla \mathbf{u}^{\varepsilon}(\tau)\right\|_{\mathbf{L}^{2}(\Omega)} d \tau+\int_{s}^{t}\left\|\mathbf{f}^{\varepsilon}(\tau)\right\|_{\mathbf{H}^{-1}(\Omega)} d \tau\right),
\end{aligned}
$$

which immediately implies that $\left\|P^{\varepsilon}(t)-P^{\varepsilon}(s)\right\|_{L^{2}(\Omega)} \rightarrow 0$ as $|t-s| \rightarrow 0$, that is, $P^{\varepsilon} \in C\left([0, T] ; L^{2}(\Omega)\right)$. The uniform estimate $(57)$ can be also deduced from (56) and Theorem 1 by choosing $\mathbf{w}^{\varepsilon}(t) \in \mathbf{H}_{0}^{1}(\Omega)$ such that $\operatorname{div} \mathbf{w}^{\varepsilon}(t)=P^{\varepsilon}(t)$ and $\left\|\mathbf{w}^{\varepsilon}(t)\right\|_{\mathbf{H}^{1}(\Omega)} \leq C|| P^{\varepsilon}(t) \|_{L^{2}(\Omega)}$. Similarly, adapting the above argument, we can show that there exists a unique $P \in C\left([0, T] ; L^{2}(\Omega) / \mathbb{R}\right)$ such that

$$
\begin{aligned}
& \left(\mathbf{u}(t)-\mathbf{u}_{0}, \mathbf{w}\right)_{\mathbf{L}^{2}(\Omega)}+\int_{0}^{t} \int_{\Omega} \xi_{\beta}^{j} \frac{\partial w_{j}}{\partial x_{\beta}} d x d \tau-(P(t), \operatorname{div} \mathbf{w})_{L^{2}(\Omega)} \\
= & \int_{0}^{t}\langle\mathbf{f}(\tau), \mathbf{w}\rangle d \tau
\end{aligned}
$$

for all $t \in[0, T]$ and $\mathbf{w} \in \mathbf{H}_{0}^{1}(\Omega)$. It follows easily from (51), (52), (56) and (59) that

$$
\left(P^{\varepsilon_{m}}, w\right)_{L^{2}(\Omega)} \rightarrow(P, w)_{L^{2}(\Omega)} \quad \text { uniformly on } \quad[0, T]
$$

for all $w \in L^{2}(\Omega)$.

Next, we introduce the adjoints $\mathbf{A}_{1}^{*}$ and $a_{Y}^{*}$ of $\mathbf{A}_{1}$ and $a_{Y}$, respectively, defined by

$$
\left(\mathbf{A}_{1}^{*} \mathbf{v}\right)_{j}=-\frac{\partial}{\partial y_{\beta}}\left(a_{\beta \alpha}^{j i}(\mathbf{y}) \frac{\partial v_{i}}{\partial y_{\alpha}}\right) \quad(j=1, \ldots, n)
$$


and

$$
a_{Y}^{*}(\mathbf{v}, \mathbf{w})=\int_{Y} a_{\delta \gamma}^{l k}(\mathbf{y}) \frac{\partial v_{k}}{\partial y_{\gamma}} \frac{\partial w_{l}}{\partial y_{\delta}} d y=a_{Y}(\mathbf{w}, \mathbf{v}) .
$$

Let us fix $\gamma, k$ with $1 \leq \gamma, k \leq n$. Then the proof of Lemma 2 can be easily adapted to deduce the existence of a unique pair $\left(\hat{\chi}_{\gamma}^{k}, \hat{q}_{\gamma}^{k}\right)$ in $\mathbf{H}_{p e r}^{1}(Y) \times L^{2}(Y)$ such that

$$
\left\{\begin{array}{cc}
\mathbf{A}_{1}^{*} \hat{\chi}_{\gamma}^{k}-\nabla \hat{q}_{\gamma}^{k}=\mathbf{A}_{1}^{*}\left(y_{\gamma} \mathbf{e}^{k}\right) & \text { in } \\
\operatorname{div} \hat{\chi}_{\gamma}^{k}=0 & \text { in } Y \\
\int_{Y} \hat{\chi}_{\gamma}^{k} d y=0, \quad \int_{Y} \hat{q}_{\gamma}^{k} d y=0 &
\end{array}\right.
$$

Note in particular that

$$
a_{Y}\left(\mathbf{v}, \hat{\chi}_{\gamma}^{k}\right)=a_{Y}\left(\mathbf{v}, y_{\gamma} \mathbf{e}^{k}\right)
$$

for all $\mathbf{v} \in \mathbf{H}_{\text {per }}^{1}$ with $\operatorname{div} \mathbf{v}=0$. For the sake of simplicity, let us denote

$$
\begin{gathered}
\hat{\chi}=\hat{\chi}_{\gamma}^{k}, \quad \hat{q}=\hat{q}_{\gamma}^{k}, \quad \hat{\mathbf{w}}=y_{\gamma} \mathbf{e}^{k}-\hat{\chi}, \quad \hat{\chi}^{\varepsilon}(\mathbf{x})=\varepsilon \hat{\chi}\left(\frac{\mathbf{x}}{\varepsilon}\right), \\
\hat{q}^{\varepsilon}(\mathbf{x})=\varepsilon \hat{q}\left(\frac{\mathbf{x}}{\varepsilon}\right), \quad \hat{\mathbf{w}}^{\varepsilon}(\mathbf{x})=\varepsilon \hat{\mathbf{w}}\left(\frac{\mathbf{x}}{\varepsilon}\right) \quad \text { and } \quad \mathbf{W}=x_{\gamma} \mathbf{e}^{k} .
\end{gathered}
$$

Then since $\hat{\chi}, \hat{q}$ are periodic and $\hat{\mathbf{w}}^{\varepsilon}=\mathbf{W}-\hat{\chi}^{\varepsilon}$, it follows from a standard result (see [5, Chapter 2] for instance) that

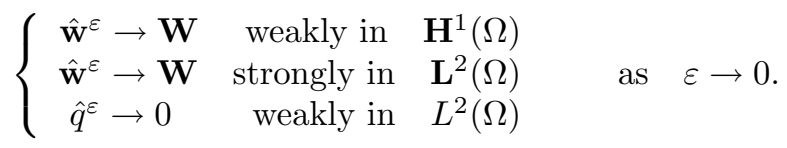

Moreover, it is easy to show (see [5, Chapter 4]) that $\left(\hat{\mathbf{w}}^{\varepsilon}, \hat{q}^{\varepsilon}\right)$ satisfies

$$
\left(\mathbf{A}^{\varepsilon}\right)^{*} \hat{\mathbf{w}}^{\varepsilon}-\nabla \hat{q}^{\varepsilon}=0 \quad \text { in } \Omega,
$$

that is,

$$
a^{\varepsilon}\left(\mathbf{v}, \hat{\mathbf{w}}^{\varepsilon}\right)+\int_{\Omega} \hat{q}^{\varepsilon} \operatorname{div} \mathbf{v} d x=0 \quad \text { for all } \quad \mathbf{v} \in \mathbf{H}_{0}^{1}(\Omega) .
$$

Now let $\varphi \in C_{c}^{\infty}(\Omega)$ be fixed. Then taking $\mathbf{v}=\varphi \hat{\mathbf{w}}^{\varepsilon_{m}}$ in (56) with $\varepsilon=\varepsilon_{m}$, we have

$$
\begin{aligned}
\left(\mathbf{u}^{\varepsilon_{m}}(t)-\mathbf{u}_{0}^{\varepsilon_{m}}, \varphi \hat{\mathbf{w}}^{\varepsilon_{m}}\right)_{\mathbf{L}^{2}(\Omega)}+\int_{0}^{t} a^{\varepsilon_{m}}\left(\mathbf{u}^{\varepsilon_{m}}(\tau), \varphi \hat{\mathbf{w}}^{\varepsilon_{m}}\right) d \tau \\
-\left(P^{\varepsilon_{m}}(t), \operatorname{div}\left(\varphi \hat{\mathbf{w}}^{\varepsilon_{m}}\right)\right)_{L^{2}(\Omega)}=\int_{0}^{t}\left\langle\mathbf{f}^{\varepsilon_{m}}(\tau), \varphi \hat{\mathbf{w}}^{\varepsilon_{m}}\right\rangle d \tau .
\end{aligned}
$$

Taking $\mathbf{v}=\varphi \mathbf{u}^{\varepsilon_{m}}(\tau)$ in (64) with $\varepsilon=\varepsilon_{m}$ and integrating over $(0, t)$, we also have

$$
\int_{0}^{t} a^{\varepsilon_{m}}\left(\varphi \mathbf{u}^{\varepsilon_{m}}(\tau), \hat{\mathbf{w}}^{\varepsilon_{m}}\right) d \tau+\int_{\Omega} \hat{q}^{\varepsilon_{m}} \operatorname{div}\left(\int_{0}^{t} \varphi \mathbf{u}^{\varepsilon_{m}}(\tau) d \tau\right) d x=0 .
$$


Subtraction of these two identities yields

$$
\begin{aligned}
& \int_{0}^{t}\left(a^{\varepsilon_{m}}\left(\mathbf{u}^{\varepsilon_{m}}(\tau), \varphi \hat{\mathbf{w}}^{\varepsilon_{m}}\right)-a^{\varepsilon_{m}}\left(\varphi \mathbf{u}^{\varepsilon_{m}}(\tau), \hat{\mathbf{w}}^{\varepsilon_{m}}\right)\right) d \tau \\
= & \int_{\Omega}\left(P^{\varepsilon_{m}}(t) \operatorname{div}\left(\varphi \hat{\mathbf{w}}^{\varepsilon_{m}}\right)+\hat{q}^{\varepsilon_{m}} \operatorname{div}\left(\int_{0}^{t} \varphi \mathbf{u}^{\varepsilon_{m}}(\tau) d \tau\right)\right) d x \\
& +\int_{0}^{t}\left\langle\mathbf{f}^{\varepsilon_{m}}(\tau), \varphi \hat{\mathbf{w}}^{\varepsilon_{m}}\right\rangle d \tau-\left(\mathbf{u}^{\varepsilon_{m}}(t)-\mathbf{u}_{0}^{\varepsilon_{m}}, \varphi \hat{\mathbf{w}}^{\varepsilon_{m}}\right)_{\mathbf{L}^{2}(\Omega)} .
\end{aligned}
$$

We calculate the limit of each term in (65) as $\varepsilon_{m} \rightarrow 0$. First, it follows immediately from (45), (52) and (63) that

$$
\left(\mathbf{u}^{\varepsilon_{m}}(t)-\mathbf{u}_{0}^{\varepsilon_{m}}, \varphi \hat{\mathbf{w}}^{\varepsilon_{m}}\right)_{\mathbf{L}^{2}(\Omega)} \rightarrow\left(\mathbf{u}(t)-\mathbf{u}_{0}, \varphi \mathbf{W}\right)_{\mathbf{L}^{2}(\Omega)}
$$

and

$$
\int_{0}^{t}\left\langle\mathbf{f}^{\varepsilon_{m}}(\tau), \varphi \hat{\mathbf{w}}^{\varepsilon_{m}}\right\rangle d \tau \rightarrow \int_{0}^{t}\langle\mathbf{f}(\tau), \varphi \mathbf{W}\rangle d \tau
$$

as $\varepsilon_{m} \rightarrow 0$ for all $t \in[0, T]$. Using (23), (45), (51), (60), (61) and (63), we obtain

$$
\begin{aligned}
& \int_{\Omega}\left(P^{\varepsilon_{m}}(t) \operatorname{div}\left(\varphi \hat{\mathbf{w}}^{\varepsilon_{m}}\right)+\hat{q}^{\varepsilon_{m}} \operatorname{div}\left(\int_{0}^{t} \varphi \mathbf{u}^{\varepsilon_{m}}(\tau) d \tau\right)\right) d x \\
= & \int_{\Omega}\left(P^{\varepsilon_{m}}(t)\left(\nabla \varphi \cdot \hat{\mathbf{w}}^{\varepsilon_{m}}+\varphi \operatorname{div} \mathbf{W}\right)+\hat{q}^{\varepsilon_{m}} \int_{0}^{t}\left(\nabla \varphi \cdot \mathbf{u}^{\varepsilon_{m}}(\tau)+\varphi g^{\varepsilon_{m}}(\tau)\right) d \tau\right) d x \\
\rightarrow & \int_{\Omega} P(t) \operatorname{div}(\varphi \mathbf{W}) d x \quad \text { as } \quad \varepsilon_{m} \rightarrow 0 \text { for all } t \in[0, T] .
\end{aligned}
$$

Finally, noting that

$$
\nabla \hat{\mathbf{w}}^{\varepsilon_{m}}(\mathbf{x})=\nabla_{\mathbf{y}} \hat{\mathbf{w}}\left(\frac{\mathbf{x}}{\varepsilon_{m}}\right) \quad \text { and } \quad \hat{\mathbf{w}} \text { is periodic }
$$

we deduce that

$$
\begin{aligned}
& \int_{0}^{t}\left(a^{\varepsilon_{m}}\left(\mathbf{u}^{\varepsilon_{m}}(\tau), \varphi \hat{\mathbf{w}}^{\varepsilon_{m}}\right)-a^{\varepsilon_{m}}\left(\varphi \mathbf{u}^{\varepsilon_{m}}(\tau), \hat{\mathbf{w}}^{\varepsilon_{m}}\right)\right) d \tau \\
= & \int_{0}^{t} \int_{\Omega} a_{\alpha \beta}^{i j}\left(\frac{\cdot}{\varepsilon_{m}}\right)\left[\frac{\partial u_{i}^{\varepsilon_{m}}}{\partial x_{\alpha}} \frac{\partial \varphi}{\partial x_{\beta}} \hat{w}_{j}^{\varepsilon_{m}}-\frac{\partial \varphi}{\partial x_{\alpha}} u_{i}^{\varepsilon_{m}} \frac{\partial \hat{w}_{j}^{\varepsilon_{m}}}{\partial x_{\beta}}\right] d x d \tau \\
= & \int_{0}^{t} \int_{\Omega}^{\varepsilon_{m}} \xi_{\beta}^{j} \frac{\partial \varphi}{\partial x_{\beta}} \hat{w}_{j}^{\varepsilon_{m}} d x d \tau-\int_{0}^{t} \int_{\Omega}\left(a_{\alpha \beta}^{i j} \frac{\partial \hat{w}_{j}}{\partial y_{\beta}}\right)\left(\frac{\cdot}{\varepsilon_{m}}\right) \frac{\partial \varphi}{\partial x_{\alpha}} u_{i}^{\varepsilon_{m}} d x d \tau \\
\rightarrow & \int_{0}^{t} \int_{\Omega} \xi_{\beta}^{j} \frac{\partial \varphi}{\partial x_{\beta}} W_{j} d x d \tau-\left(\int_{Y} a_{\alpha \beta}^{i j} \frac{\partial \hat{w}_{j}}{\partial y_{\beta}} d y\right)\left(\int_{0}^{t} \int_{\Omega} \frac{\partial \varphi}{\partial x_{\alpha}} u_{i} d x d \tau\right)
\end{aligned}
$$


as $\varepsilon_{m} \rightarrow 0$ for all $t \in[0, T]$. Hence letting $\varepsilon_{m} \rightarrow 0$ in (65), we have

$$
\begin{aligned}
& \int_{0}^{t} \int_{\Omega} \xi_{\beta}^{j} \frac{\partial \varphi}{\partial x_{\beta}} W_{j} d x d \tau-\left(\int_{Y} a_{\alpha \beta}^{i j} \frac{\partial \hat{w}_{j}}{\partial y_{\beta}} d y\right)\left(\int_{0}^{t} \int_{\Omega} \frac{\partial \varphi}{\partial x_{\alpha}} u_{i} d x d \tau\right) \\
= & \int_{\Omega} P(t) \operatorname{div}(\varphi \mathbf{W}) d x+\int_{0}^{t}\langle\mathbf{f}(\tau), \varphi \mathbf{W}\rangle d \tau-\left(\mathbf{u}(t)-\mathbf{u}_{0}, \varphi \mathbf{W}\right)_{\mathbf{L}^{2}(\Omega)} .
\end{aligned}
$$

Therefore, combining this and (59) with $\mathbf{w}=\varphi \mathbf{W}$ taken, we conclude that

$$
\int_{0}^{t} \int_{\Omega} \xi_{\beta}^{j} \frac{\partial W_{j}}{\partial x_{\beta}} \varphi d x d \tau=-\left(\int_{Y} a_{\alpha \beta}^{i j} \frac{\partial \hat{w}_{j}}{\partial y_{\beta}} d y\right)\left(\int_{0}^{t} \int_{\Omega} \frac{\partial \varphi}{\partial x_{\alpha}} u_{i} d x d \tau\right)
$$

for all $t \in[0, T]$ and $\varphi \in C_{c}^{\infty}(\Omega)$, which implies that

$$
\xi_{\beta}^{j} \frac{\partial W_{j}}{\partial x_{\beta}}=\left(\int_{Y} a_{\alpha \beta}^{i j} \frac{\partial \hat{w}_{j}}{\partial y_{\beta}} d y\right) \frac{\partial u_{i}}{\partial x_{\alpha}} \quad \text { in } \quad \Omega \times(0, T) .
$$

Then recalling that $\mathbf{W}=x_{\gamma} \mathbf{e}^{k}$ and $\hat{\mathbf{w}}=y_{\gamma} \mathbf{e}^{k}-\hat{\chi}_{\gamma}^{k}$, we immediately obtain

$$
\xi_{\gamma}^{k}=\left(\int_{Y} a_{\alpha \beta}^{i j} \frac{\partial}{\partial y_{\beta}}\left(y_{\gamma} e_{j}^{k}-\left(\hat{\chi}_{\gamma}^{k}\right)_{j}\right) d y\right) \frac{\partial u_{i}}{\partial x_{\alpha}}
$$

and replacing the indices, we have

$$
\xi_{\beta}^{j}=\left(\int_{Y} a_{\alpha \delta}^{i l} \frac{\partial}{\partial y_{\delta}}\left(y_{\beta} e_{l}^{j}-\left(\hat{\chi}_{\beta}^{j}\right)_{l}\right) d y\right) \frac{\partial u_{i}}{\partial x_{\alpha}} .
$$

Hence to prove (55), it remains to show that

$$
\tilde{a}_{\alpha, \beta}^{i j}=\int_{Y}\left(a_{\alpha \beta}^{i j}-a_{\alpha \delta}^{i l} \frac{\partial}{\partial y_{\delta}}\left(\hat{\chi}_{\beta}^{j}\right)_{l}\right) d y .
$$

But taking $\mathbf{w}=\hat{\chi}_{\beta}^{j}$ in (40) and $\mathbf{v}=\chi_{\alpha}^{i}$ in (62), we have

$$
a_{Y}\left(y_{\alpha} \mathbf{e}^{i}, \hat{\chi}_{\beta}^{j}\right)=a_{Y}\left(\chi_{\alpha}^{i}, \hat{\chi}_{\beta}^{j}\right)=a_{Y}\left(\chi_{\alpha}^{i}, y_{\beta} \mathbf{e}^{j}\right) .
$$

Using this result together with (34) and (39), we derive (66). We have completed the proof of Theorem 7 .

\section{The corrector theorem}

A formal asymptotic expansion of $\mathbf{u}^{\varepsilon}$ was derived in Section 3. Up to the first order of $\varepsilon$, we have

$$
\mathbf{u}^{\varepsilon}(\mathbf{x}, t)=\mathbf{u}^{0}(\mathbf{x}, t)-\varepsilon \chi_{\gamma}^{k}\left(\frac{\mathbf{x}}{\varepsilon}\right) \frac{\partial u_{k}^{0}}{\partial x_{\gamma}}(\mathbf{x}, t)+\cdots .
$$

Recall from Definition 3 that each pair $(\chi, q)=\left(\chi_{\gamma}^{k}, q_{\gamma}^{k}\right)$ is the unique weak solution in $\mathbf{H}_{\text {per }}^{1}(Y) \times L_{\text {per }}^{2}(Y)$ to the problem

$$
\left\{\begin{array}{cc}
\mathbf{A}^{1} \chi+\nabla q=\mathbf{A}^{1}\left(y_{\gamma} \mathbf{e}^{k}\right) & \text { in } \quad Y \\
\operatorname{div} \chi=0 & \text { in } Y \\
\int_{Y} \chi d y=0, \quad \int_{Y} q d y= & 0
\end{array}\right.
$$


In particular, since $\left(\chi_{\gamma}^{k}, q_{\gamma}^{k}\right) \in \mathbf{H}_{p e r}^{1}(Y) \times L^{2}(Y)$, we easily deduce from a standard result (see [5] for instance) that

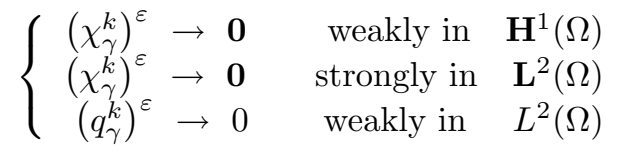

as $\varepsilon \rightarrow 0$, where

$$
\left(\chi_{\gamma}^{k}\right)^{\varepsilon}(\mathbf{x})=\varepsilon \chi_{\gamma}^{k}\left(\frac{\mathbf{x}}{\varepsilon}\right) \quad \text { and } \quad\left(q_{\gamma}^{k}\right)^{\varepsilon}(\mathbf{x})=\varepsilon q_{\gamma}^{k}\left(\frac{\mathbf{x}}{\varepsilon}\right) .
$$

Hence it follows from the homogenization theorem, Theorem 7 , that

$$
\mathbf{u}^{\varepsilon}+\left(\chi_{\gamma}^{k}\right)^{\varepsilon} \frac{\partial u_{k}^{0}}{\partial x_{\gamma}} \rightarrow \mathbf{u}^{0} \quad \text { weakly in } \quad L^{2}\left(0, T ; \mathbf{H}^{1}(\Omega)\right)
$$

as $\varepsilon \rightarrow 0$, provided that $\mathbf{u}^{0}$ is sufficiently regular. However, this convergence turns out to be strong as shown in the following corrector theorem.

Theorem 9. Assume in addition to the hypotheses of Theorem 7 that

(70) $\quad \mathbf{G}^{\varepsilon} \rightarrow \mathbf{G}^{0} \quad$ strongly in $\quad \mathbf{W}_{T} \quad$ and $\quad \mathbf{u}_{0}^{\varepsilon} \rightarrow \mathbf{u}_{0}^{0} \quad$ strongly in $\mathbf{L}^{2}(\Omega)$.

Then it follows that

$$
\mathbf{u}^{\varepsilon} \rightarrow \mathbf{u}^{0} \quad \text { strongly in } \quad C\left([0, T] ; \mathbf{L}^{2}(\Omega)\right) .
$$

Moreover, if the homogenized limit $\mathbf{u}_{0}$ has the additional regularity

$$
\mathbf{u}^{0} \in L^{2}\left(0, T ; \mathbf{W}^{2, n}(\Omega)\right),
$$

then we have

$$
\mathbf{u}^{\varepsilon}+\left(\chi_{\gamma}^{k}\right)^{\varepsilon} \frac{\partial u_{k}^{0}}{\partial x_{\gamma}} \rightarrow \mathbf{u}^{0} \quad \text { strongly in } \quad L^{2}\left(0, T ; \mathbf{H}^{1}(\Omega)\right) .
$$

Remark 10. Since $\mathbf{u}^{0}$ is a weak solution to the linear parabolic problem $\left(H P^{0}\right)$ with constant coefficients, the additional regularity (71) of $\mathbf{u}^{0}$ can be obtained by assuming higher regularity of the data $\mathbf{f}^{0}, g^{0}, \mathbf{u}_{b}^{0}$ and $\mathbf{u}_{0}^{0}$.

To prove Theorem 9, we follow a general approach based on the previous homogenization theorem and energy identities. See [4], [5] and [6]. First we introduce the corrector operator $\mathbf{C}^{\varepsilon}$ defined by

$$
\mathbf{C}^{\varepsilon}(\mathbf{x}) \Phi=\Phi-\nabla\left(\chi_{\gamma}^{k}\right)^{\varepsilon}(\mathbf{x}) \Phi_{\gamma}^{k}=\Phi-\nabla_{\mathbf{y}} \chi_{\gamma}^{k}\left(\frac{\mathbf{x}}{\varepsilon}\right) \Phi_{\gamma}^{k}
$$

for all $n \times n$-matrices $\Phi=\left(\Phi_{\gamma}^{k}\right)$. We can rewrite $\mathbf{C}^{\varepsilon}$ as

$$
\mathbf{C}^{\varepsilon}(\mathbf{x}) \Phi=\nabla\left(\mathbf{w}_{\gamma}^{k}\right)^{\varepsilon}(\mathbf{x}) \Phi_{\gamma}^{k}=\nabla_{\mathbf{y}} \mathbf{w}_{\gamma}^{k}\left(\frac{\mathbf{x}}{\varepsilon}\right) \Phi_{\gamma}^{k}
$$

where

$$
\mathbf{w}_{\gamma}^{k}(\mathbf{y})=y_{\gamma} \mathbf{e}^{k}-\chi_{\gamma}^{k}(\mathbf{y}) \text { and }\left(\mathbf{w}_{\gamma}^{k}\right)^{\varepsilon}(\mathbf{x})=\varepsilon \mathbf{w}_{\gamma}^{k}\left(\frac{\mathbf{x}}{\varepsilon}\right)=x_{\gamma} \mathbf{e}^{k}-\left(\chi_{\gamma}^{k}\right)^{\varepsilon}(\mathbf{x}) .
$$

The crucial steps of our proof of Theorem 9 are to prove the following two lemmas. 
Lemma 11. For each $\gamma, k$ with $1 \leq \gamma, k \leq n$, the pair $\left(\left(\mathbf{w}_{\gamma}^{k}\right)^{\varepsilon},\left(q_{\gamma}^{k}\right)^{\varepsilon}\right)$ satisfies

$$
\begin{aligned}
& \mathbf{A}^{\varepsilon}\left(\mathbf{w}_{\gamma}^{k}\right)^{\varepsilon}+\nabla\left(q_{\gamma}^{k}\right)^{\varepsilon}=0, \quad \operatorname{div}\left(\mathbf{w}_{\gamma}^{k}\right)^{\varepsilon}=\operatorname{div}\left(x_{\gamma} \mathbf{e}^{k}\right) \quad \text { in } \Omega, \\
& \left(\left(\mathbf{w}_{\gamma}^{k}\right)^{\varepsilon},\left(q_{\gamma}^{k}\right)^{\varepsilon}\right) \in \mathbf{W}^{1, r}(\Omega) \times L^{r}(\Omega) \quad \text { for some } \quad r \in(2, \infty)
\end{aligned}
$$

and

$$
\left(\left(\mathbf{w}_{\gamma}^{k}\right)^{\varepsilon},\left(q_{\gamma}^{k}\right)^{\varepsilon}\right) \rightarrow\left(x_{\gamma} \mathbf{e}^{k}, 0\right) \quad \text { weakly in } \quad \mathbf{W}^{1, r}(\Omega) \times L^{r}(\Omega)
$$

Lemma 12. Let the conditions (45) and (70) be satisfied by the data. For each fixed matrix-valued function

$$
\Phi=\left(\Phi_{\alpha}^{i}\right) \quad \text { with } \Phi_{\alpha}^{i} \in C_{c}^{\infty}(\Omega \times(0, T)) \text { for each } \alpha, i,
$$

we define a function $\rho^{\varepsilon}=\rho_{\Phi}^{\varepsilon}$ on $[0, T]$ by

$$
\begin{aligned}
\rho^{\varepsilon}(t)= & \frac{1}{2}\left\|\mathbf{v}^{\varepsilon}(t)-\mathbf{v}^{0}(t)\right\|_{\mathbf{L}^{2}(\Omega)}^{2} \\
& +\int_{0}^{t} \int_{\Omega} a_{\alpha \beta}^{i j}(\dot{\bar{\varepsilon}})\left(\frac{\partial u_{i}^{\varepsilon}}{\partial x_{\alpha}}-\left(\mathbf{C}^{\varepsilon} \Phi\right)_{\alpha}^{i}\right)\left(\frac{\partial u_{j}^{\varepsilon}}{\partial x_{\beta}}-\left(\mathbf{C}^{\varepsilon} \Phi\right)_{\beta}^{j}\right) d x d \tau
\end{aligned}
$$

for all $t \in[0, T]$, where $\mathbf{v}^{\varepsilon}=\mathbf{u}^{\varepsilon}-\mathbf{G}^{\varepsilon}$ and $\mathbf{v}^{0}=\mathbf{u}^{0}-\mathbf{G}^{0}$ as before.

Then we have

$$
\rho^{\varepsilon} \rightarrow \rho^{0} \quad \text { strongly in } \quad C([0, T]) \text {, }
$$

where

$$
\rho^{0}(t)=\rho_{\Phi}^{0}(t)=\int_{0}^{t} \int_{\Omega} \tilde{a}_{\alpha \beta}^{i j}\left(\frac{\partial u_{i}^{0}}{\partial x_{\alpha}}-\Phi_{\alpha}^{i}\right)\left(\frac{\partial u_{j}^{0}}{\partial x_{\beta}}-\Phi_{\beta}^{j}\right) d x d \tau
$$

for all $t \in[0, T]$.

Assuming the validity of two lemmas, we first prove Theorem 9.

Proof of Theorem 9. Let $\eta>0$ be a small fixed number. Then since $\nabla \mathbf{u}^{0} \in$ $L^{2}\left(0, T ; \mathbf{L}^{2}(\Omega)\right)$, there exists a matrix-valued function $\Phi \in\left[C_{c}^{\infty}(\Omega \times(0, T))\right]^{n}$ such that $\left\|\nabla \mathbf{u}^{0}-\Phi\right\|_{L^{2}\left(0, T ; \mathbf{L}^{2}(\Omega)\right)} \leq \eta$. Let $\rho_{\Phi}^{\varepsilon}$ and $\rho_{\Phi}^{0}$ be the functions defined in Lemma 12. Then it follows from (4) and (18) that

$$
\begin{aligned}
& \left\|\mathbf{u}^{\varepsilon}-\mathbf{u}^{0}\right\|_{C\left([0, T] ; \mathbf{L}^{2}(\Omega)\right)}^{2}+\left\|\operatorname{Sym}\left(\nabla \mathbf{u}^{\varepsilon}-\mathbf{C}^{\varepsilon} \Phi\right)\right\|_{L^{2}\left(0, T ; \mathbf{L}^{2}(\Omega)\right)}^{2} \\
\leq & C\left\|\mathbf{G}^{\varepsilon}-\mathbf{G}^{0}\right\|_{C\left([0, T] ; \mathbf{L}^{2}(\Omega)\right)}^{2}+C\left\|\rho_{\Phi}^{\varepsilon}\right\|_{L^{\infty}(0, T)} \\
\leq & C\left\|\mathbf{G}^{\varepsilon}-\mathbf{G}^{0}\right\|_{C\left([0, T] ; \mathbf{L}^{2}(\Omega)\right)}^{2}+C\left\|\rho_{\Phi}^{\varepsilon}-\rho_{\Phi}^{0}\right\|_{L^{\infty}(0, T)}+C \eta^{2} .
\end{aligned}
$$

Here for an $n \times n$-matrix $\xi=\left(\xi_{\alpha}^{i}\right)_{1<\alpha, i<n}$, we denote by $\operatorname{Sym}(\xi)$ the symmetric part of $\xi: \operatorname{Sym}(\xi)=\left(\frac{1}{2}\left(\xi_{\alpha}^{i}+\xi_{i}^{\alpha}\right)\right)_{1 \leq \alpha, i \leq n}$. Hence using (70) and Lemma 12, we have

$$
\limsup _{\varepsilon \rightarrow 0}\left\|\mathbf{u}^{\varepsilon}-\mathbf{u}^{0}\right\|_{C\left([0, T] ; \mathbf{L}^{2}(\Omega)\right)} \leq C \eta,
$$

which implies that

$$
\lim _{\varepsilon \rightarrow 0}\left\|\mathbf{u}^{\varepsilon}-\mathbf{u}^{0}\right\|_{C\left([0, T] ; \mathbf{L}^{2}(\Omega)\right)}=0
$$


because $\eta>0$ is arbitrary.

Assume further that $\mathbf{u}^{0} \in L^{2}\left(0, T ; \mathbf{W}^{2, n}(\Omega)\right)$. Then since

$$
\nabla \mathbf{u}^{0} \in L^{2}\left(0, T ; \mathbf{W}^{1, n}(\Omega)\right) \hookrightarrow L^{2}\left(0, T ; \mathbf{L}^{2 r /(r-2)}(\Omega)\right),
$$

the function $\Phi \in\left[C_{c}^{\infty}(\Omega \times(0, T))\right]^{n}$ can be chosen so that

$$
\left\|\nabla \mathbf{u}^{0}-\Phi\right\|_{L^{2}\left(0, T ; \mathbf{L}^{2 r /(r-2)}(\Omega)\right)} \leq \eta .
$$

Recall from Lemma 11 that $\left\{\nabla\left(\mathbf{w}_{\gamma}^{k}\right)^{\varepsilon}\right\}_{\varepsilon>0}$ is a bounded set in $\mathbf{L}^{r}(\Omega)$. Hence using (74), we have

$$
\begin{aligned}
& \left\|\operatorname{Sym}\left(\nabla \mathbf{u}^{\varepsilon}-\mathbf{C}^{\varepsilon} \nabla \mathbf{u}^{0}\right)\right\|_{L^{2}\left(0, T ; \mathbf{L}^{2}(\Omega)\right)} \\
\leq & \left\|\operatorname{Sym}\left(\nabla \mathbf{u}^{\varepsilon}-\mathbf{C}^{\varepsilon} \Phi\right)\right\|_{L^{2}\left(0, T ; \mathbf{L}^{2}(\Omega)\right)}+\left\|\mathbf{C}^{\varepsilon} \Phi-\mathbf{C}^{\varepsilon} \nabla \mathbf{u}^{0}\right\|_{L^{2}\left(0, T ; \mathbf{L}^{2}(\Omega)\right)} \\
\leq & \left\|\operatorname{Sym}\left(\nabla \mathbf{u}^{\varepsilon}-\mathbf{C}^{\varepsilon} \Phi\right)\right\|_{L^{2}\left(0, T ; \mathbf{L}^{2}(\Omega)\right)}+C \eta .
\end{aligned}
$$

Combining (70), (72), (75) and Lemma 12, we deduce that

$$
\limsup _{\varepsilon \rightarrow 0}\left\|\operatorname{Sym}\left(\nabla \mathbf{u}^{\varepsilon}-\mathbf{C}^{\varepsilon} \nabla \mathbf{u}^{0}\right)\right\|_{L^{2}\left(0, T ; \mathbf{L}^{2}(\Omega)\right)} \leq C \eta
$$

for any $\eta>0$ and thus

$$
\lim _{\varepsilon \rightarrow 0}\left\|\operatorname{Sym}\left(\nabla \mathbf{u}^{\varepsilon}-\nabla \mathbf{u}^{0}+\nabla\left(\chi_{\gamma}^{k}\right)^{\varepsilon} \frac{\partial u_{k}^{0}}{\partial x_{\gamma}}\right)\right\|_{L^{2}\left(0, T ; \mathbf{L}^{2}(\Omega)\right)}=0 .
$$

On the other hand, it follows from (71) and Lemma 11 that

$$
\left(\chi_{\gamma}^{k}\right)^{\varepsilon} \rightarrow 0 \quad \text { in } \quad \mathbf{L}^{\frac{2 n}{n-2}}(\Omega) \quad \text { and } \quad\left|\left(\chi_{\gamma}^{k}\right)^{\varepsilon}\right|\left|\nabla \frac{\partial u_{k}^{0}}{\partial x_{\gamma}}\right| \rightarrow 0 \quad \text { in } \quad L^{2}\left(0, T ; \mathbf{L}^{2}(\Omega)\right) \text {. }
$$

Using these results together with Theorem 7 and (76), we easily show that

$$
\left.\lim _{\varepsilon \rightarrow 0}\left\|\mathbf{u}^{\varepsilon}-\mathbf{u}^{0}+\left(\chi_{\gamma}^{k}\right)^{\varepsilon} \frac{\partial u_{k}^{0}}{\partial x_{\gamma}}\right\|\right|_{L^{2}\left(0, T ; \mathbf{L}^{2}(\Omega)\right)}=0
$$

and

$$
\lim _{\varepsilon \rightarrow 0}\left\|\operatorname{Sym} \nabla\left(\mathbf{u}^{\varepsilon}-\mathbf{u}^{0}+\left(\chi_{\gamma}^{k}\right)^{\varepsilon} \frac{\partial u_{k}^{0}}{\partial x_{\gamma}}\right)\right\|_{L^{2}\left(0, T ; \mathbf{L}^{2}(\Omega)\right)}=0 .
$$

Theorem 9 follows immediately from (73), (77), (78) and classical Korn's inequality

$$
\|\mathbf{v}\|_{\mathbf{H}^{1}(\Omega)} \leq C\left(\|\mathbf{v}\|_{\mathbf{L}^{2}(\Omega)}+\|\operatorname{Sym} \nabla \mathbf{v}\|_{\mathbf{L}^{2}(\Omega)}\right) \quad \text { for all } \quad \mathbf{v} \in \mathbf{H}^{1}(\Omega) ;
$$

see [11] or [14] for a proof.

To complete the proof of Theorem 9, it remains to prove the two key lemmas, Lemmas 11 and 12. We first provide a detailed proof of Lemma 12.

Proof of Lemma 12. Let $\Phi \in\left[C_{c}^{\infty}(\Omega \times(0, T))\right]^{n}$ be fixed, and we write

$$
\rho^{\varepsilon}=\rho_{1}^{\varepsilon}+\rho_{2}^{\varepsilon}+\rho_{3}^{\varepsilon}+\rho_{4}^{\varepsilon} \quad(\varepsilon>0),
$$


where

$$
\begin{aligned}
& \rho_{1}^{\varepsilon}(t)=\frac{1}{2}\left\|\mathbf{v}^{\varepsilon}(t)-\mathbf{v}^{0}(t)\right\|_{\mathbf{L}^{2}(\Omega)}+\int_{0}^{t} a^{\varepsilon}\left(\mathbf{u}^{\varepsilon}(\tau), \mathbf{u}^{\varepsilon}(\tau)\right) d \tau \\
& \rho_{2}^{\varepsilon}(t)=-\int_{0}^{t} \int_{\Omega} a_{\alpha \beta}^{i j}\left(\frac{\cdot}{\varepsilon}\right) \frac{\partial u_{i}^{\varepsilon}}{\partial x_{\alpha}}\left(\mathbf{C}^{\varepsilon} \Phi\right)_{\beta}^{j} d x d \tau \\
& \rho_{3}^{\varepsilon}(t)=-\int_{0}^{t} \int_{\Omega} a_{\alpha \beta}^{i j}\left(\frac{\cdot}{\varepsilon}\right)\left(\mathbf{C}^{\varepsilon} \Phi\right)_{\alpha}^{i} \frac{\partial u_{j}^{\varepsilon}}{\partial x_{\beta}} d x d \tau
\end{aligned}
$$

and

$$
\rho_{4}^{\varepsilon}(t)=\int_{0}^{t} \int_{\Omega} a_{\alpha \beta}^{i j}\left(\frac{\cdot}{\varepsilon}\right)\left(\mathbf{C}^{\varepsilon} \Phi\right)_{\alpha}^{i}\left(\mathbf{C}^{\varepsilon} \Phi\right)_{\beta}^{j} d x d \tau .
$$

We calculate the limit of each $\rho_{l}^{\varepsilon}$ as $\varepsilon \rightarrow 0$. To calculate $\lim _{\varepsilon \rightarrow 0} \rho_{1}^{\varepsilon}$, we will make use of the following energy identity

$$
\begin{aligned}
& \left\|\mathbf{v}^{\varepsilon}(t)\right\|_{\mathbf{L}^{2}(\Omega)}^{2}+2 \int_{0}^{t} a^{\varepsilon}\left(\mathbf{v}^{\varepsilon}(\tau), \mathbf{v}^{\varepsilon}(\tau)\right) d \tau \\
= & \left\|\mathbf{v}^{\varepsilon}(0)\right\|_{\mathbf{L}^{2}(\Omega)}^{2}+2 \int_{0}^{t}\left\langle\tilde{\mathbf{f}}^{\varepsilon}(\tau), \mathbf{v}^{\varepsilon}(\tau)\right\rangle d \tau,
\end{aligned}
$$

where $\tilde{\mathbf{f}}^{\varepsilon}=\mathbf{f}^{\varepsilon}-\partial_{t} \mathbf{G}^{\varepsilon}-\mathbf{A}^{\varepsilon} \mathbf{G}^{\varepsilon} \in L^{2}\left(0, T ; \mathbf{H}^{-1}(\Omega)\right)$. It should be noted that the energy identity (79) holds for all $\varepsilon \in[0, \infty)$ and $t \in[0, T]$. From (79), it also follows that

$$
\begin{aligned}
& \left\|\mathbf{v}^{\varepsilon}(t)\right\|_{\mathbf{L}^{2}(\Omega)}^{2}+2 \int_{0}^{t} a^{\varepsilon}\left(\mathbf{u}^{\varepsilon}(\tau), \mathbf{v}^{\varepsilon}(\tau)\right) d \tau \\
= & \left\|\mathbf{v}^{\varepsilon}(0)\right\|_{\mathbf{L}^{2}(\Omega)}^{2}+2 \int_{0}^{t}\left\langle\left(\mathbf{f}^{\varepsilon}-\partial_{t} \mathbf{G}^{\varepsilon}\right)(\tau), \mathbf{v}^{\varepsilon}(\tau)\right\rangle d \tau
\end{aligned}
$$

for all $\varepsilon \in[0, \infty)$ and $t \in[0, T]$. Hence $\rho_{1}^{\varepsilon}$ can be rewritten as

$$
\begin{aligned}
\rho_{1}^{\varepsilon}(t)= & \frac{1}{2}\left\|\mathbf{v}^{\varepsilon}(t)-\mathbf{v}^{0}(t)\right\|_{\mathbf{L}^{2}(\Omega)}+\int_{0}^{t} a^{\varepsilon}\left(\mathbf{u}^{\varepsilon}(\tau),\left(\mathbf{v}^{\varepsilon}+\mathbf{G}^{\varepsilon}\right)(\tau)\right) d \tau \\
= & \frac{1}{2}\left\|\mathbf{v}^{\varepsilon}(0)\right\|_{\mathbf{L}^{2}(\Omega)}-\left(\mathbf{v}^{\varepsilon}(t), \mathbf{v}^{0}(t)\right)_{\mathbf{L}^{2}(\Omega)}+\frac{1}{2}\left\|\mathbf{v}^{0}(t)\right\|_{\mathbf{L}^{2}(\Omega)} \\
& +\int_{0}^{t} a^{\varepsilon}\left(\mathbf{u}^{\varepsilon}(\tau), \mathbf{G}^{\varepsilon}(\tau)\right) d \tau+\int_{0}^{t}\left\langle\left(\mathbf{f}^{\varepsilon}-\partial_{t} \mathbf{G}^{\varepsilon}\right)(\tau), \mathbf{v}^{\varepsilon}(\tau)\right\rangle d \tau
\end{aligned}
$$

and by virtue of (45), (46), (48), (70) and (80) with $\varepsilon=0$, we obtain

$$
\begin{aligned}
\lim _{\varepsilon \rightarrow 0} \rho_{1}^{\varepsilon}(t)= & \frac{1}{2}\left\|\mathbf{v}^{0}(0)\right\|_{\mathbf{L}^{2}(\Omega)}-\frac{1}{2}\left\|\mathbf{v}^{0}(t)\right\|_{\mathbf{L}^{2}(\Omega)}+\int_{0}^{t} a^{0}\left(\mathbf{u}^{0}(\tau), \mathbf{G}^{0}(\tau)\right) d \tau \\
& +\int_{0}^{t}\left\langle\left(\mathbf{f}^{0}-\partial_{t} \mathbf{G}^{0}\right)(\tau), \mathbf{v}^{0}(\tau)\right\rangle d \tau \\
= & \int_{0}^{t} a^{0}\left(\mathbf{u}^{0}(\tau), \mathbf{u}^{0}(\tau)\right) d \tau \quad \text { for all } \quad t \in[0, T] .
\end{aligned}
$$


To calculate $\lim _{\varepsilon \rightarrow 0} \rho_{2}^{\varepsilon}$, we write

$$
\begin{aligned}
\rho_{2}^{\varepsilon}(t) & =-\int_{0}^{t} \int_{\Omega} a_{\alpha \beta}^{i j}\left(\frac{\cdot}{\varepsilon}\right) \frac{\partial u_{i}^{\varepsilon}}{\partial x_{\alpha}}\left[\frac{\partial}{\partial x_{\beta}}\left(\left(\mathbf{w}_{\gamma}^{k}\right)_{j}^{\varepsilon} \Phi_{\gamma}^{k}\right)-\left(\mathbf{w}_{\gamma}^{k}\right)_{j}^{\varepsilon} \frac{\partial \Phi_{\gamma}^{k}}{\partial x_{\beta}}\right] d x d \tau \\
& =-\int_{0}^{t} a^{\varepsilon}\left(\mathbf{u}^{\varepsilon},\left(\mathbf{w}_{\gamma}^{k}\right)^{\varepsilon} \Phi_{\gamma}^{k}\right) d \tau+\int_{0}^{t} \int_{\Omega} a_{\alpha \beta}^{i j}\left(\frac{\cdot}{\varepsilon}\right) \frac{\partial u_{i}^{\varepsilon}}{\partial x_{\alpha}}\left(\mathbf{w}_{\gamma}^{k}\right)_{j}^{\varepsilon} \frac{\partial \Phi_{\gamma}^{k}}{\partial x_{\beta}} d x d \tau .
\end{aligned}
$$

Using (56), (46), (48), (60), Lemma 11 and then (59), we have

$$
\begin{aligned}
& \int_{0}^{t} a^{\varepsilon}\left(\mathbf{u}^{\varepsilon},\left(\mathbf{w}_{\gamma}^{k}\right)^{\varepsilon} \Phi_{\gamma}^{k}\right) d \tau \\
= & \left(P^{\varepsilon}(t), \operatorname{div}\left(\left(\mathbf{w}_{\gamma}^{k}\right)^{\varepsilon} \Phi_{\gamma}^{k}\right)\right)_{L^{2}(\Omega)}+\int_{0}^{t}\left\langle\mathbf{f}^{\varepsilon},\left(\mathbf{w}_{\gamma}^{k}\right)^{\varepsilon} \Phi_{\gamma}^{k}\right\rangle d \tau \\
& -\left(\mathbf{u}^{\varepsilon}(t)-\mathbf{u}^{\varepsilon}(0),\left(\mathbf{w}_{\gamma}^{k}\right)^{\varepsilon} \Phi_{\gamma}^{k}\right)_{\mathbf{L}^{2}(\Omega)} \\
\rightarrow & \left(P^{0}(t), \operatorname{div}\left(x_{\gamma} \mathbf{e}^{k} \Phi_{\gamma}^{k}\right)\right)_{L^{2}(\Omega)}+\int_{0}^{t}\left\langle\mathbf{f}^{0}, x_{\gamma} \mathbf{e}^{k} \Phi_{\gamma}^{k}\right\rangle d \tau \\
& -\left(\mathbf{u}^{0}(t)-\mathbf{u}^{0}(0), x_{\gamma} \mathbf{e}^{k} \Phi_{\gamma}^{k}\right)_{\mathbf{L}^{2}(\Omega)} \\
= & \int_{0}^{t} a^{0}\left(\mathbf{u}^{0}, x_{\gamma} \mathbf{e}^{k} \Phi_{\gamma}^{k}\right) d \tau=\int_{0}^{t} \int_{\Omega} \tilde{a}_{\alpha \beta}^{i j} \frac{\partial u_{i}^{0}}{\partial x_{\alpha}} \frac{\partial\left(x_{\gamma} e_{j}^{k} \Phi_{\gamma}^{k}\right)}{\partial x_{\beta}} d x d \tau
\end{aligned}
$$

and

$$
\int_{0}^{t} \int_{\Omega} a_{\alpha \beta}^{i j}\left(\frac{\cdot}{\varepsilon}\right) \frac{\partial u_{i}^{\varepsilon}}{\partial x_{\alpha}}\left(\mathbf{w}_{\gamma}^{k}\right)_{j}^{\varepsilon} \frac{\partial \Phi_{\gamma}^{k}}{\partial x_{\beta}} d x d \tau \rightarrow \int_{0}^{t} \int_{\Omega} \tilde{a}_{\alpha \beta}^{i j} \frac{\partial u_{i}^{0}}{\partial x_{\alpha}}\left(x_{\gamma} e_{j}^{k}\right) \frac{\partial \Phi_{\gamma}^{k}}{\partial x_{\beta}} d x d \tau
$$

so that

$$
\begin{aligned}
\lim _{\varepsilon \rightarrow 0} \rho_{2}^{\varepsilon}(t) & =-\int_{0}^{t} \int_{\Omega} \tilde{a}_{\alpha \beta}^{i j} \frac{\partial u_{i}^{0}}{\partial x_{\alpha}} \frac{\partial\left(x_{\gamma} e_{j}^{k}\right)}{\partial x_{\beta}} \Phi_{\gamma}^{k} d x d \tau \\
& =-\int_{0}^{t} \int_{\Omega} \tilde{a}_{\alpha \beta}^{i j} \frac{\partial u_{i}^{0}}{\partial x_{\alpha}} \Phi_{\beta}^{j} d x d \tau \quad \text { for all } t \in[0, T] .
\end{aligned}
$$

Using Lemma 11, (23), (47) and then (39), we can also calculate $\lim _{\varepsilon \rightarrow 0} \rho_{3}^{\varepsilon}$ as follows:

$$
\begin{aligned}
\rho_{3}^{\varepsilon}(t) & =-\int_{0}^{t} \int_{\Omega} a_{\alpha \beta}^{i j}\left(\frac{\dot{\varepsilon}}{\varepsilon}\right) \frac{\partial}{\partial x_{\alpha}}\left(\mathbf{w}_{\gamma}^{k}\right)_{i}^{\varepsilon}\left[\frac{\partial}{\partial x_{\beta}}\left(\Phi_{\gamma}^{k} u_{j}^{\varepsilon}\right)-u_{j}^{\varepsilon} \frac{\partial \Phi_{\gamma}^{k}}{\partial x_{\beta}}\right] d x d \tau \\
& =-\int_{0}^{t} a^{\varepsilon}\left(\left(\mathbf{w}_{\gamma}^{k}\right)^{\varepsilon}, \Phi_{\gamma}^{k} \mathbf{u}^{\varepsilon}\right) d \tau+\int_{0}^{t} \int_{\Omega} a_{\alpha \beta}^{i j}\left(\frac{\dot{-}}{\varepsilon}\right) \frac{\partial}{\partial x_{\alpha}}\left(\mathbf{w}_{\gamma}^{k}\right)_{i}^{\varepsilon} u_{j}^{\varepsilon} \frac{\partial \Phi_{\gamma}^{k}}{\partial x_{\beta}} d x d \tau \\
& =-\int_{0}^{t} \int_{\Omega}\left(q_{\gamma}^{k}\right)^{\varepsilon} \operatorname{div}\left(\Phi_{\gamma}^{k} \mathbf{u}^{\varepsilon}\right) d x d \tau+\int_{0}^{t} \int_{\Omega}\left(a_{\alpha \beta}^{i j} \frac{\partial}{\partial y_{\alpha}}\left(\mathbf{w}_{\gamma}^{k}\right)_{i}\right)\left(\frac{\dot{-}}{\varepsilon}\right) u_{j}^{\varepsilon} \frac{\partial \Phi_{\gamma}^{k}}{\partial x_{\beta}} d x d \tau \\
\rightarrow & \left(\int_{Y} a_{\alpha \beta}^{i j} \frac{\partial}{\partial y_{\alpha}}\left(\mathbf{w}_{\gamma}^{k}\right)_{i} d y\right)\left(\int_{0}^{t} \int_{\Omega} u_{j}^{0} \frac{\partial \Phi_{\gamma}^{k}}{\partial x_{\beta}} d x d \tau\right)=\int_{0}^{t} \int_{\Omega} \tilde{a}_{\gamma \beta}^{k j} u_{j}^{0} \frac{\partial \Phi_{\gamma}^{k}}{\partial x_{\beta}} d x d \tau
\end{aligned}
$$


and thus

$$
\lim _{\varepsilon \rightarrow 0} \rho_{3}^{\varepsilon}(t)=-\int_{0}^{t} \int_{\Omega} \tilde{a}_{\alpha \beta}^{i j} \Phi_{\alpha}^{i} \frac{\partial u_{j}^{0}}{\partial x_{\beta}} d x d \tau \quad \text { for all } t \in[0, T] .
$$

A similar calculation yields

$$
\begin{aligned}
\rho_{4}^{\varepsilon}(t)= & \int_{0}^{t} a^{\varepsilon}\left(\left(\mathbf{w}_{\gamma}^{k}\right)^{\varepsilon},\left(\mathbf{w}_{\delta}^{l}\right)^{\varepsilon} \Phi_{\gamma}^{k} \Phi_{\delta}^{l}\right) d \tau \\
& -\int_{0}^{t} \int_{\Omega}\left(a_{\alpha \beta}^{i j} \frac{\partial}{\partial y_{\alpha}}\left(\mathbf{w}_{\gamma}^{k}\right)_{i}\right)\left(\frac{\cdot}{\varepsilon}\right)\left(\mathbf{w}_{\delta}^{l}\right)_{j}^{\varepsilon} \frac{\partial}{\partial x_{\beta}}\left(\Phi_{\gamma}^{k} \Phi_{\delta}^{l}\right) d x d \tau \\
\rightarrow & -\int_{0}^{t} \int_{\Omega} \tilde{a}_{\gamma \beta}^{k j}\left(x_{\delta} e_{j}^{l}\right) \frac{\partial}{\partial x_{\beta}}\left(\Phi_{\gamma}^{k} \Phi_{\delta}^{l}\right) d x d \tau
\end{aligned}
$$

and so

$$
\lim _{\varepsilon \rightarrow 0} \rho_{4}^{\varepsilon}(t)=\int_{0}^{t} \int_{\Omega} \tilde{a}_{\alpha \beta}^{i j} \Phi_{\alpha}^{i} \Phi_{\beta}^{j} d x d \tau \quad \text { for all } t \in[0, T]
$$

Combining (82)-(85), we have shown that

$$
\lim _{\varepsilon \rightarrow 0} \rho^{\varepsilon}(t)=\rho^{0}(t) \quad \text { for all } \quad t \in[0, T] .
$$

We now show that $\left\{\rho^{\varepsilon}\right\}$ actually converges uniformly on $[0, T]$. For this purpose, we have only to show that for every sequence $\left\{\varepsilon_{m}\right\}$ with $\varepsilon_{m} \rightarrow 0$, the sequence $\left\{\rho^{\varepsilon_{m}}\right\}_{m \in \mathbf{N}}$ converges uniformly on $[0, T]$ as $m \rightarrow \infty$. But since the pointwise convergence of $\left\{\rho^{\varepsilon_{m}}\right\}$ was already shown, it suffices to show, by the AscoliArzelà theorem, that the family $\left\{\rho^{\varepsilon_{m}}\right\}_{m \in \mathbf{N}}$ is equicontinuous on $[0, T]$. Suppose that $0 \leq t<t+h \leq T$. Then from (81) with $\varepsilon=\varepsilon_{m}$, we deduce that

$$
\begin{aligned}
& \left|\rho_{1}^{\varepsilon_{m}}(t+h)-\rho_{1}^{\varepsilon_{m}}(t)\right| \\
\leq & \left.\int_{t}^{t+h}\left|2 \frac{d}{d \tau}\left(\mathbf{v}^{\varepsilon_{m}}, \mathbf{v}^{0}\right)_{\mathbf{L}^{2}(\Omega)}+\frac{d}{d \tau} \| \mathbf{v}^{0}\right|\right|_{\mathbf{L}^{2}(\Omega)} ^{2} \mid d \tau \\
& +2 \int_{t}^{t+h}\left|a^{\varepsilon_{m}}\left(\mathbf{u}^{\varepsilon_{m}}(\tau), \mathbf{G}^{\varepsilon_{m}}(\tau)\right)+\left\langle\left(\mathbf{f}^{\varepsilon_{m}}-\partial_{t} \mathbf{G}^{\varepsilon_{m}}\right)(\tau), \mathbf{v}^{\varepsilon_{m}}(\tau)\right\rangle\right| d \tau .
\end{aligned}
$$

Recall from (11) that

$$
\frac{d}{d t}\left(\mathbf{v}^{\varepsilon_{m}}(\tau), \mathbf{v}^{0}(\tau)\right)_{\mathbf{L}^{2}(\Omega)}=\left\langle\partial_{t} \mathbf{v}^{\varepsilon_{m}}(\tau), \mathbf{v}^{0}(\tau)\right\rangle+\left\langle\partial_{t} \mathbf{v}^{0}(\tau), \mathbf{v}^{\varepsilon_{m}}(\tau)\right\rangle
$$

for a.e. $\tau \in[0, T]$. Moreover, since

$$
\left\|\mathbf{v}^{\varepsilon}\right\|_{\mathbf{v}_{T}}+\left\|\mathbf{u}^{\varepsilon}\right\|_{L^{2}\left(0, T ; \mathbf{H}^{1}(\Omega)\right)} \leq C
$$


for some constant $C$ independent of $\varepsilon \geq 0$, it easily follows that

$$
\begin{aligned}
& \left|\rho_{1}^{\varepsilon_{m}}(t+h)-\rho_{1}^{\varepsilon_{m}}(t)\right| \\
\leq & C\left(\int_{t}^{t+h}\left(\left\|\mathbf{v}^{0}\right\|_{V}^{2}+\left\|\partial_{t} \mathbf{v}^{0}\right\|_{V^{\prime}}^{2}\right) d \tau\right)^{\frac{1}{2}}+C\left(\int_{t}^{t+h}\left\|\mathbf{G}^{\varepsilon_{m}}\right\|_{\mathbf{H}^{1}(\Omega)}^{2} d \tau\right)^{\frac{1}{2}} \\
& +C\left(\int_{t}^{t+h}\left\|\partial_{t} \mathbf{G}^{\varepsilon_{m}}\right\|_{\mathbf{H}^{-1}(\Omega)}^{2} d \tau\right)^{\frac{1}{2}}+C\left(\int_{t}^{t+h}\left\|\mathbf{f}^{\varepsilon_{m}}\right\|_{\mathbf{H}^{-1}(\Omega)}^{2} d \tau\right)^{\frac{1}{2}} .
\end{aligned}
$$

This implies the equicontinuity of $\left\{\rho_{1}^{\varepsilon_{m}}\right\}_{m \in \mathbf{N}}$ on $[0, T]$ because $\mathbf{v}^{0} \in \mathbf{V}_{T}$, $\mathbf{G}^{\varepsilon_{m}} \rightarrow \mathbf{G}^{0}$ strongly in $\mathbf{W}_{T}$ and $\mathbf{f}^{\varepsilon_{m}} \rightarrow \mathbf{f}^{0}$ strongly in $L^{2}\left(0, T ; \mathbf{H}^{-1}(\Omega)\right)$. On the other hand, by virtue of (86) and Lemma 11, we deduce that

$$
\begin{aligned}
\left|\rho_{2}^{\varepsilon}(t+h)-\rho_{2}^{\varepsilon}(t)\right| & \leq \int_{t}^{t+h}\left|\int_{\Omega} a_{\alpha \beta}^{i j}\left(\frac{\dot{-}}{\varepsilon}\right) \frac{\partial u_{i}^{\varepsilon}}{\partial x_{\alpha}}\left(\mathbf{C}^{\varepsilon} \Phi\right)_{\beta}^{j}\right| d x d \tau \\
& \leq C \int_{t}^{t+h}\left\|\nabla \mathbf{u}^{\varepsilon}(\tau)\right\|_{\mathbf{L}^{2}(\Omega)}\left\|\nabla\left(\mathbf{w}_{\gamma}^{k}\right)^{\varepsilon} \Phi_{\gamma}^{k}(\tau)\right\|_{\mathbf{L}^{2}(\Omega)} d \tau \\
& \leq C(\Phi) h^{\frac{1}{2}}
\end{aligned}
$$

and similarly

$$
\left|\rho_{3}^{\varepsilon}(t+h)-\rho_{3}^{\varepsilon}(t)\right|+\left|\rho_{4}^{\varepsilon}(t+h)-\rho_{4}^{\varepsilon}(t)\right| \leq C(\Phi)\left(h^{\frac{1}{2}}+h\right),
$$

which implies the equicontinuity of each of the families $\left\{\rho_{2}^{\varepsilon}\right\}_{\varepsilon>0},\left\{\rho_{3}^{\varepsilon}\right\}_{\varepsilon>0}$ and $\left\{\rho_{4}^{\varepsilon}\right\}_{\varepsilon>0}$. This completes the proof of Lemma 12 .

Proof of Lemma 11. The first assertion can be easily deduced from (68). Suppose next that there is some $r>2$ such that $\left(\mathbf{w}_{\gamma}^{k}, q_{\gamma}^{k}\right) \in \mathbf{W}^{1, r}(Y) \times L^{r}(Y)$. Then since $\chi_{\gamma}^{k}, q_{\gamma}^{k}$ are periodic and $\mathbf{w}_{\gamma}^{k}(\mathbf{y})=y_{\gamma} \mathbf{e}^{k}-\chi_{\gamma}^{k}(\mathbf{y})$, it follows from a standard result (see [5, Chapter 2] for instance) that $\left\{\left(\left(\mathbf{w}_{\gamma}^{k}\right)^{\varepsilon},\left(q_{\gamma}^{k}\right)^{\varepsilon}\right)\right\}_{\varepsilon>0}$ is a weakly convergent sequence in $\mathbf{W}^{1, r}(\Omega) \times L^{r}(\Omega)$. Hence the second and third assertions are immediate consequences of (69). Therefore, to complete the proof of Lemma 11, it suffices to show that if $(\mathbf{w}, q) \in \mathbf{H}^{1}\left(Q_{2}\right) \times L^{2}\left(Q_{2}\right)$ is a weak solution of the Stokes equations

$$
\mathbf{A}_{1} \mathbf{w}-\nabla q=0 \quad \text { in } \quad Q_{2} \quad \text { and } \quad \operatorname{div} \mathbf{w}=g \in L^{\infty}\left(Q_{2}\right),
$$

where $Q_{2}=(-2,2)^{n} \subset \mathbb{R}^{n}$, then $(\mathbf{w}, q) \in \mathbf{W}^{1, r}(Y) \times L^{r}(Y)$ for some $r>2$. This is an extension to the Stokes equations of a classical $L^{r}$-regularity result due to Meyers [10] for general elliptic equations with $L^{\infty}$-coefficients; see [2] for a quite simple proof. Here we provide a complete, different proof based on the reverse Hölder inequality.

Suppose that $\mathbf{x}_{0} \in Q_{2}$ and $B_{2 R}=B_{2 R}\left(\mathbf{x}_{0}\right)=\left\{\mathbf{x} \in \mathbb{R}^{n}:\left|\mathbf{x}-\mathbf{x}_{0}\right|<2 R\right\} \subset Q_{2}$, and let us denote

$$
\overline{\mathbf{w}}=\frac{1}{\left|B_{2 R}\right|} \int_{B_{2 R}} \mathbf{w} d x \quad \text { and } \quad \bar{q}=\frac{1}{\left|B_{2 R}\right|} \int_{B_{2 R}} q d x .
$$


We first show that if $\nabla \mathbf{w} \in \mathbf{L}^{s}\left(B_{2 R}\right)$ and $1<s<\infty$, then $q \in L^{s}\left(B_{2 R}\right)$ and

$$
\|q-\bar{q}\|_{L^{s}\left(B_{2 R}\right)} \leq C\|\nabla \mathbf{w}\|_{\mathbf{L}^{s}\left(B_{2 R}\right)}
$$

for some positive constant $C=C(n, s, M, \lambda)$, independent of $R$. For this, let $\psi \in L^{\infty}\left(B_{2 R}\right)$ be fixed. Then by virtue of Bogovskii's result (see [3] and [7]), there exist a vector field $\mathbf{h}$ in $\mathbf{W}^{1, s /(s-1)}(\Omega) \cap \mathbf{H}_{0}^{1}\left(B_{2 R}\right)$ and a positive constant $C$, independent of $R$, such that

$$
\operatorname{div} \mathbf{h}=\psi-\bar{\psi} \quad \text { in } \quad B_{2 R} \quad \text { and } \quad\|\nabla \mathbf{h}\|_{\mathbf{L}^{\frac{s}{s-1}}\left(B_{2 R}\right)} \leq C\|\psi-\bar{\psi}\|_{L^{\frac{s}{s-1}}\left(B_{2 R}\right)},
$$

where $\bar{\psi}=\frac{1}{\left|B_{2 R}\right|} \int_{B_{2 R}} \psi d x$. Taking $\mathbf{h}$ as a test function in the weak formulation of (87), we deduce that

$$
\begin{aligned}
\int_{B_{2 R}}(q-\bar{q}) \psi d x & =\int_{B_{2 R}}(q-\bar{q}) \operatorname{div} \mathbf{h} d x \\
& \leq C \int_{B_{2 R}}|\nabla \mathbf{w} \| \nabla \mathbf{h}| d x \\
& \leq C\|\nabla \mathbf{w}\|_{L^{s}\left(B_{2 R}\right)}|| \nabla \mathbf{h} \|_{\mathbf{L}^{\frac{s}{s-1}}\left(B_{2 R}\right)} \\
& \leq C\|\nabla \mathbf{w}\|_{L^{s}\left(B_{2 R}\right)}\|\psi-\bar{\psi}\|_{L^{\frac{s}{s-1}\left(B_{2 R}\right)}},
\end{aligned}
$$

which proves $(88)$ because $\psi \in L^{\infty}\left(B_{2 R}\right)$ is arbitrary and

$$
\|\bar{\psi}\|_{L^{\frac{s}{s-1}}\left(B_{2 R}\right)} \leq\|\psi\|_{L^{\frac{s}{s-1}}\left(B_{2 R}\right)} .
$$

Hence to complete the proof of the lemma, it suffices to show that $\mathbf{w} \in$ $\mathbf{W}^{1, r}\left(Q_{1}\right)$ for some $r>2$. To show this, let $\varphi \in C_{c}^{\infty}\left(B_{2 R}\right)$ be a smooth cut-off function such that $0 \leq \varphi \leq 1,|\nabla \varphi| \leq \frac{C}{R}$ in $B_{2 R}$ and $\varphi=1$ in $B_{R}$. Then denoting

$$
\mathbf{v}=(\mathbf{w}-\overline{\mathbf{w}}) \varphi \in \mathbf{H}_{0}^{1}\left(Q_{2}\right)
$$

and taking $\varphi \mathbf{v}$ as a test function in the weak formulation of (87), we deduce that

$$
\int a_{\alpha \beta}^{i j} \frac{\partial w_{i}}{\partial x_{\alpha}}\left(\frac{\partial w_{j}}{\partial x_{\beta}} \varphi^{2}+2 v_{j} \varphi \frac{\partial \varphi}{\partial x_{\beta}}\right) d x=\int(q-\bar{q})\left(g \varphi^{2}+2 \mathbf{v} \cdot \nabla \varphi\right) d x .
$$

By a simple calculation, we have

$$
\begin{gathered}
\int a_{\alpha \beta}^{i j} \frac{\partial v_{i}}{\partial x_{\alpha}} \frac{\partial v_{j}}{\partial x_{\beta}} d x \leq C \int|\nabla \mathbf{w}||\mathbf{w}-\overline{\mathbf{w}}||\nabla \varphi|+|\mathbf{w}-\overline{\mathbf{w}}|^{2}|\nabla \varphi|^{2} d x \\
+\int|q-\bar{q}|(|g|+|\mathbf{w}-\overline{\mathbf{w}}||\nabla \varphi|) d x
\end{gathered}
$$

and

$$
\begin{aligned}
\int_{B_{R}}|\nabla \mathbf{w}|^{2} d x \leq & \frac{C}{R} \int_{B_{2 R}}|\nabla \mathbf{w}||\mathbf{w}-\overline{\mathbf{w}}| d x+\frac{C}{R^{2}} \int_{B_{2 R}}|\mathbf{w}-\overline{\mathbf{w}}|^{2} d x \\
& +C \int_{B_{2 R}}|q-\bar{q}||g| d x+\frac{C}{R} \int_{B_{2 R}}|q-\bar{q}||\mathbf{w}-\overline{\mathbf{w}}| d x
\end{aligned}
$$


for some constant $C$ independent of $R$. In view of Young's inequality and the pressure estimate (88), we also have

$$
\int_{B_{R}}|\nabla \mathbf{w}|^{2} d x \leq C_{\theta}\left(\int_{B_{2 R}}|g|^{2} d x+\frac{1}{R^{2}} \int_{B_{2 R}}|\mathbf{w}-\overline{\mathbf{w}}|^{2} d x\right)+\theta \int_{B_{2 R}}|\nabla \mathbf{w}|^{2} d x
$$

for any small $\theta>0$. Hence using Poincaré-Sobolev inequality, we deduce that

$\int_{B_{R}}|\nabla \mathbf{w}|^{2} d x \leq C_{\theta}\left[\int_{B_{2 R}}|g|^{2} d x+\frac{1}{R^{2}}\left(\int_{B_{2 R}}|\nabla \mathbf{w}|^{\frac{2 n}{n+2}} d x\right)^{\frac{n+2}{n}}\right]+\theta \int_{B_{2 R}}|\nabla \mathbf{w}|^{2} d x$

for any small $\theta>0$. Therefore, applying a $\theta$-version of the reverse Hölder inequality (see [1] for instance), we conclude that $\mathbf{w} \in \mathbf{W}^{1, r}\left(Q_{1}\right)$ for some $r>2$. One can also remove the $\theta$-term in (89) using a standard covering argument and then apply original Gehring's result [8] to draw the same conclusion. See [9] for details. This completes the proof of Lemma 11.

\section{References}

[1] A. Bensoussan and J. Frehse, Regularity Results for Nonlinear Elliptic Systems and Applications, Applied Mathematical Sciences 151, Springer-Verlag, Berlin, 2002.

[2] A. Bensoussan, J. L. Lions, and G. Papanicolaou, Asymptotic Analysis for Periodic Structures, North-Holland, Amsterdam, 1978.

[3] M. E. Bogovskii, Solution of the first boundary value problem for an equation of continuity of an incompressible medium, Dokl. Akad. Nauk SSSR 248 (1979), no. 5, 1037-1040. (Russian); English Transl.: Soviet Math Dokl. 20 (1979), 1094-1098.

[4] S. Brahim-Stsmane, G. A. Francfort, and F. Murat, Correctors for the homogenization of the wave and heat equations, J. Math. Pures Appl. (9) 71 (1992), no. 3, 197-231.

[5] D. Cioranescu and P. Donato, An Introduction to Homogenization, Oxford Lecture Series in Mathematics and its Applications, 17. The Clarendon Press, Oxford University Press, New York, 1999.

[6] C. Conca, On the application of the homogenization theory to a class of problems arising in fluid mechanics, J. Math. Pures Appl. (9) 64 (1985), no. 1, 31-75.

[7] G. P. Galdi, An introduction to the mathematical theory of the Navier-Stokes equations. Vol. I, Springer Tracts in Natural Philosophy, 38. Springer-Verlag, New York, 1994.

[8] F. W. Gehring, The $L^{p}$-integrability of the partial derivatives of a quasiconformal mapping, Acta Math. 130 (1973), 265-277.

[9] M. Giaquinta and G. Modica, Nonlinear systems of the type of the stationary NavierStokes system, J. Reine Angew. Math. 330 (1982), 173-214.

[10] N. G. Meyers, An $L^{p}$ e-estimate for the gradient of solutions of second order elliptic divergence equations, Ann. Scuola Norm. Sup. Pisa (3) 17 (1963), 189-206.

[11] J. Nečas and I. Hlavaček, Mathematical Theory of Elastic and Elasto-Plastic Bodies: An Introduction, Studies in Applied Mechanics 3, Elsevier Scientific Publishing Co., Amsterdam-New York, 1980.

[12] E. Sánchez-Palencia, Nonhomogeneous Media and Vibration Theory, Lecture Notes in Phys. 127, Springer-Verlag, Berlin, 1980.

[13] R. Temam, Navier-Stokes Equations: Theory and Numerical Analysis, North-Holland, Amsterdam, 1984.

[14] L. Wang, On Korn's inequality, J. Comput. Math. 21 (2003), no. 3, 321-324. 
Hi Jun ChOE

Department of Mathematics

YONSEI UNIVERSITY

SEOUl 120-749, KoreA

E-mail address: choe@yonsei.ac.kr

HYUNSEOK KIM

Department of Mathematics

SOGANG UNIVERSITY

SEOUl 121-742, KoreA

E-mail address: khs319@kias.re.kr 